\title{
Data Retrieval Algorithms for Validating the Optical Transient Detector and the Lightning Imaging Sensor
}

\author{
W. J. Koshak and R. J. Blakeslee
}

Global Hydrology and Climate Center, NASA Marshall Space Flight Center, Huntsville, Alabama

\author{
J. C. BAILEY
}

Raytheon STX, Global Hydrolngy and Climate Center, NASA Marshall Space Fligh Center, Huntsville, Alabama

(Manuscript received 4 February 1999, in final form 1 June 1999)

\section{ABSTRACT}

A linear algebraic solution is provided for the problem of retrieving the location and time of occurrence of lightning ground strikes from an Advanced Lightning Direction Finder (ALDF) network. The ALDF network measures field strength, magnetic bearing, and arrival time of lightning radio cmissions. Solutions for the plane (i.c., no carth curvature) are provided that implement all of these measurements. The accuracy of the retrieval method is tested using computer-simulated datasets, and the relative influence of bearing and arrival time data on the outcome of the final solution is formally demonstrated. The algorithm is sufficiently accurate to validate NASA's Optical Transient Detector and Lightning Imaging Sensor. A quadratic planar solution that is useful when only three arrival time measurements are available is also introduced. The algebra of the quadratic root results are examined in detail to clarify what portions of the analysis region lead to fundamental ambiguities in source location. Complex root results are shown to be associated with the presence of measurement errors when the lightning source lies near an outer sensor baseline of the ALDF network. For arbitrary noncollinear network geometries and in the absence of measurement errors, it is shown that the two quadratic roots are equivalent (no source location ambiguity) on the outer sensor baselines. The accuracy of the guadratic planar method is tested with computer-generated datasets, and the results are generally better than those obtained from the threestation linear planar method when bearing errors are about $2^{\circ}$.

\section{Introduction}

Advanced Lightning Direction Finder (ALDF) sensors, developed by Global Atmospherics Inc. (GAI), have the ability to detect the field strength, magnetic bearing, and arrival time of lightning radio emissions. In 1992, Lightning Location and Protection, Inc. (a division of GAI) completed development of an Improved Performance from Combined Technology (IMPACT) method for determining the location and time of occurrence of lightning return strokes from these data (Cummins et al. 1993). The IMPACT algorithm is based on minimizing a $\chi^{2}$ function similar to that provided in Eq. (1) of Hiscox et al. (1984) but generalized to accommodate arrival time data. The lightning time of occurrence $t$, the longitude $\lambda$, and the latitude $\varphi$ of the lightning source on an ellipsoidal earth is estimated.

Since the IMPACT algorithm uses a numerical ap-

Corresponding author address: Dr. William J. Koshak, NASAMSFC/HR20, Global Hydrology and Climate Center, 977 Explorer Blyd., Huntsville, AL 35806.

E-mail: william.koshak@msfc.nasa.gov proach to determine the absolute minimum of the nonlinear $\chi^{2}$ hypersurface, it does not represent an analytic solution to the problem; that is, the source location and time of occurrence are not directly determined in terms of the measurements and measuring network geometry. Instead, the algorithm uses the computer to search for the optimum values of $(\lambda, \varphi, t)$ that minimize $\chi^{2}$; each new set of measurements implies starting an entirely new search for an answer. In effect, the actual solution is estimated using the constraints of the data and the power of the computer.

Relative multiple minima in the $\chi^{2}(\lambda, \varphi, t)$ hypersurface can lead to a premature termination in the computer search and an erroneous solution. The presence of data errors can generate additional relative minima and additional solution errors. Since it is computationally expensive to check for erroneous solutions during realtime processing of ALDF data, lightning source solutions depend, in general, on where in the solution space $(\lambda, \varphi, t)$ the computer search begins.

Although the IMPACT algorithm has had successful practical application (Cummings et al. 1998), the specific algorithm software is proprietary and is not widely distributed free of charge to the scientific community. 
In this study, our interest was to develop an economical, yet useful (four-station) ALDF ground-truth site at the Melville Island-Darwin, Australia, region-and elsewhere in the world-that could be used to validate the National Aeronautics and Space Administration's (NASA's) space-based lighting detectors [the Lightning Imaging Sensor (LIS), and the Optical Transient Detector (OTD) described in Christian et al. (1992) and Goodman et al. (1995), respectively]. We desired a computationally quick algorithm that easily ingests all types of ALDF measurements, and that produces groundstrike locations with accuracies better than $4 \mathrm{~km}$ (the nadir resolution of LIS) for sources that are within a few hundred kilometers of the ALDF network. This has been the primary motivation behind our algorithm development. As such, we did not require an algorithm that accounts for earth curvature.

A secondary motivation behind this work was realized during our algorithm development. We learned that a serious pedagogical effort to examine the mathematical foundations of lightning ground-strike retrievals does not appear in the literature. Perhaps the wide use and practicality of powerful numerical computer algorithms have offset the desire to obtain analytic solutions. In retrospect, the proper mathematical approach is to first solve the least difficult form of the retrieval problem in a thorough way, and then methodically upgrade the formalism to account for additional physical complexities such as associated with the addition of new datasets, earth curvature, and wave propagation effects. An effort must also be made to clearly explain fundamental ambiguities that arise in the retrieval process when an experimenter has limited measurements available due to sensor failure or other causes. Although there have been some analytic efforts to account for earth sphericity for magnetic bearing data inversions (e.g., see Orville 1987) a complete pedagogy that ultimately leads to rigorous retrievals of ground strikes on an ellipsoidal earth surface (and that uses all ALDF data constraints in a coherent/simultaneous and optimum fashion) is notably absent in the literature.

To the best of the authors' knowledge, no one has yet explicitly provided an analytic solution to the problem of determining lightning source location and time of occurrence using collective measurements of field, magnetic bearing, and arrival time measurements when earth curvature and propagation effects are neglected. This is a fundamental starting point. For multiple dataset inversions, the relative importance of bearing and arrival time data on the outcome of the final solution has not been formally demonstrated. Furthermore, no thorough investigation of solution ambiguities have been provided when one is limited to just three arrival time measurements. This writing introduces theoretical derivations that address each of these problems. In so doing, we arrive at the required OTD-LIS ground truth algorithms.

We specifically determine the source location $(x, y)$ as a mathematical function of the measurements under a variety of conditions (i.e., differences in the number, location, and type of measurements). Since algebraic solutions are obtained, we do not need to invoke a computer search algorithm to determine optimum solution parameters. We provide unique physical insight into the nature of the retrieval problem because we determine exactly how the measurements are specifically related to the lightning source location (and time of occurrence).

A Linear Planar (LP) method is first introduced that allows one to simultaneously analyze field, bearing, and arrival time measurements, and a means for optimally weighing the bearing data relative to arrival time data is demonstrated. To the best of our knowledge, the linear approach provided here has not been attempted elsewhere in the literature. The method involves one large system of linear equations that offers a high degree of flexibility from the point of view of the user's applicational needs. For example, if only a certain number and type of measurements are available in an experiment, the linear system of equations degenerates into a smaller set of equations, and a straightforward solution process is retained.

We also introduce a Quadratic Planar (QP) method that can be used when only three arrival time measurements are available. Such a situation arises if there are sensor hardware failures and/or when field amplitude and bearing measurement data quality is unacceptable. Although this method is mathematically nonlinear, full analytic solutions are derived. Physical insight about the nonlinear solution space, not discernible from conventional $\chi^{2}$ analyses, is fully described by examining in detail all quadratic root solutions derived from the QP method. For example, we show explicitly that a certain mathematical discriminant vanishes for certain lightning locations, and that these source locations produce complex roots (negative discriminants) in the presence of measurement errors.

Extensive tests of the LP and QP retrieval methods are provided using computer-simulated datasets and these methods are applied in a study of ALDF data that were obtained from the Maritime Continent Thunderstorm Experiment (MCTEX) analysis region in Darwin, Australia (Keenan et al. 1994, 1996). Data from this network comprise one of several ground-truth sites for the validation of OTD and LIS.

\section{Linear Planar (LP) method}

We begin by considering $n \geq 3$ sensors situated at locations $\mathbf{r}_{i}, i=1,2, \ldots, n$ relative to some origin. Each sensor has the capability to measure the arrival time $t_{i}$, magnetic bearing $\phi_{i}$, and field strength $F$, of the radio emissions from a lightning source with location, $\mathbf{r}$, time of occurrence, $t$, and radiation source strength, $s$. Hence, from the $3 n$ measurements $\left\{\left(t_{1}, \phi_{1}\right.\right.$, $\left.\left.F_{1}\right), \ldots,\left(t_{n}, \phi_{n}, F_{n}\right)\right\}$ we wish to determine the five 


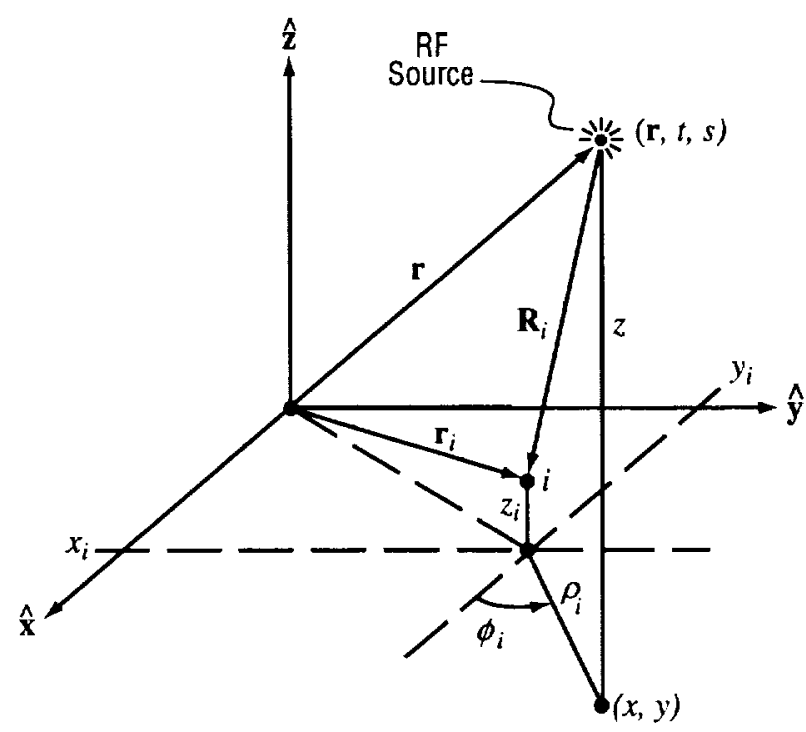

Fig. 1. Geometry associated with the LP method

unknowns $(x, y, z, t, s)$. In so doing, we neglect earth curvature

Figure 1 summarizes the geometry of the LP model. Because ALDF sensors might not be deployed on a flat topography, the $i$ th sensor located at $\mathbf{r}_{i}$ need not lie in the $x y$ plane, that is, $z_{i} \neq 0$ in general. The relative position vector follows standard physics convention, that is, it points from the source at $\mathbf{r}$ to the observation point $\mathbf{r}_{i}$, so that $\mathbf{R}_{i}=\mathbf{r}_{i}-\mathbf{r}$. Neglecting refractive effects in the atmosphere, the excitation time of the $i$ th sensor is

$$
t_{i}=t+\frac{1}{c} R_{i}
$$

where $c$ is the speed of light. Solving for $R_{i}$, squaring, and rearranging terms leads to

$$
\begin{aligned}
\frac{1}{2}\left(r_{i}^{2}-c^{2} t_{i}^{2}\right)= & x_{i} x+y_{i} y+z_{i} z-c^{2} t_{i} t \\
& -\frac{1}{2}\left(r^{2}-c^{2} t^{2}\right) .
\end{aligned}
$$

It is desirable to remove the last term on the right-hand side of (2) since it is nonlinear in the space and time variables. To do this, we define the measurement

$$
\alpha_{i} \equiv \frac{1}{2}\left(r_{i}^{2}-c^{2} t_{i}^{2}\right)-\frac{1}{2}\left(r_{i}^{2}-c^{2} t_{i}^{2}\right) .
$$

A comparison of (2) and (3) shows that $\alpha_{i}$ is linearly related to the lightning location, $\mathbf{r}=(x, y, z)$, and lightning time of occurrence, $t$; that is,

$$
\begin{aligned}
\alpha_{i}= & \left(x_{i}-x_{1}\right) x+\left(y_{i}-y_{1}\right) y+\left(z_{i}-z_{1}\right) z \\
& -c^{2}\left(t_{i}-t_{1}\right) t ; \quad i=2,3, \ldots, n .
\end{aligned}
$$

A detailed investigation of this linear form has been provided in Koshak and Solakiewicz (1996).

Next, we consider the information content of ALDF bearing data. From Fig. 1 we see that the lightning location $(x, y)$ is given by

$$
x=x_{i}+\rho_{i} \cos \phi_{i} \quad y=y_{i}+\rho_{i} \sin \phi_{i},
$$

where $\rho_{i}$ is the horizontal distance from the $i$ th site to the lightning ground-strike location. It is useful to define the measurement

$$
\beta_{i} \equiv x_{i} \sin \phi_{i}-y_{i} \cos \phi_{i} .
$$

Solving (5) for $x_{i}$ and $y_{i}$ and substituting into (6) gives

$$
\beta_{i}=\left(\sin \phi_{i}\right) x-\left(\cos \phi_{i}\right) y .
$$

Finally, we consider measurements of the radiated field strength. Assuming a $1 / R$, attenuation in the radiation field gives

$$
F_{i}=\frac{1}{R} s .
$$

Once again, we solve for $R_{1}$, square, rearrange terms, and define the measurement

$$
\gamma_{i} \equiv \frac{1}{2}\left(r_{i}^{2}-r_{i}^{2}\right)
$$

This leads to the following relation:

$$
\begin{aligned}
\gamma_{i}= & \left(x_{1}-x_{1}\right) x+\left(y_{i}-y_{1}\right) y+\left(z_{i}-z_{1}\right) z \\
& +\frac{1}{2}\left(\frac{1}{F_{i}^{2}}-\frac{1}{F_{1}^{2}}\right) s^{2} ; \quad i=2,3, \ldots, n .
\end{aligned}
$$

If we consider only $n=3$ sensors, (4), (7), and (10) can be combined to give

$$
\left[\begin{array}{c}
\alpha_{2} \\
\alpha_{3} \\
\delta \beta_{1} \\
\delta \beta_{2} \\
\delta \beta_{3} \\
\gamma_{2} \\
\gamma_{3}
\end{array}\right]=\left[\begin{array}{ccccc}
\left(x_{2}-x_{1}\right) & \left(y_{2}-y_{1}\right) & \left(z_{2}-z_{1}\right) & c\left(t_{1}-t_{2}\right) & 0 \\
\left(x_{3}-x_{1}\right) & \left(y_{3}-y_{1}\right) & \left(z_{3}-z_{1}\right) & c\left(t_{1}-t_{3}\right) & 0 \\
\delta \sin \phi_{1} & -\delta \cos \phi_{1} & 0 & 0 & 0 \\
\delta \sin \phi_{2} & -\delta \cos \phi_{2} & 0 & 0 & 0 \\
\delta \sin \phi_{3} & -\delta \cos \phi_{3} & 0 & 0 & 0 \\
\left(x_{2}-x_{1}\right) & \left(y_{2}-y_{1}\right) & \left(z_{2}-z_{1}\right) & 0 & \psi_{2}\left(x_{2}-x_{1}\right) \\
\left(x_{3}-x_{1}\right) & \left(y_{3}-y_{1}\right) & \left(z_{3}-z_{1}\right) & 0 & \psi_{3}\left(x_{2}-x_{1}\right)
\end{array}\right]\left[\begin{array}{c}
x \\
y \\
z \\
d_{1} \\
d_{s}
\end{array}\right],
$$


where $\delta$ is a weighting factor chosen as $10^{3} \mathrm{~m}, \Psi_{i}=$ $\frac{1}{2}\left[\left(F_{1} / F_{t}\right)^{2}-1\right]$ is a dimensionless parameter, $d_{t}=c t$, $d_{s}=\xi^{2} s^{2}$, and $\xi=\left[F_{1}\left(x_{2}-x_{1}\right)^{1 / 2}\right]$ is a scaling factor to be described below. Defining the column vector on the left-hand side of (11) as $\mathbf{g}$, the matrix by $\mathbf{K}$, and the remaining vector by $f$, we may rewrite (11) as

$$
\mathbf{g}=\mathbf{K f} \text {. }
$$

All elements of $\mathbf{K}$ and $\mathbf{f}$ are in units of meters, and all elements of $\mathbf{g}$ are in squared meters. This was accomplished by retaining a factor of $c$ in front of the time-difference measurements in the first two rows (fourth column) of $\mathbf{K}$, by multiplying (7) by the weighting factor $\delta$, and by scaling the field $F_{i}$ and source strength $s$, each by the factor $\xi$; that is, by making the substitutions $F_{1} \rightarrow \xi F_{1}$, and $s \rightarrow \xi s$ in (8). (Note that the sites must be numbered in such a way that $x_{2}>x_{1}$ so $\xi$ is not complex; this can always be accomplished since the numbering of sites is arbitrary and because the translation and rotation of the $x-y$ coordinate system used in the LP method is arbitrary.)

In general, the $\mathbf{K}$ matrix has $3 n-2$ rows and 5 columns, where $n=1,2,3, \ldots$ If there is only $n=$ 1 sensors in the network, $K$ degenerates into a row vector and (12) is underdetermined. If there are $n=2$ sensors, $K$ will have 4 rows and (12) will still be underdetermined. For $n \geq 3$ sites, $K$ will have $\geq 7$ rows and (12) will be overdetermined. For overdetermined systems, $f$ can be retrieved using the least squares inversion provided in Twomey (1977):

$$
\mathbf{f}=(\overline{\mathbf{K}} \mathbf{K})^{-1} \overline{\mathbf{K}} \mathbf{g} \text {, }
$$

where the tilde represents matrix transposition. The source time of occurrence and source strength are determined as $t=d_{t} / c, s=d_{s}^{1 / 2} / \xi$, respectively.

From the foregoing generalities, we now note that ALDF sensors trigger on the initial upward current surge that occurs close to the ground level in the return stroke [see, e.g., the transmission line model discussed in Uman et al. (1973)]. Hence, the source can be regarded as being located at $z=0$. In this case, we can remove the third component of $f$, that is, we consider the column vector $\mathbf{f} \equiv \operatorname{col}\left(x, y, d_{t}, d_{\mathrm{s}}\right)$, and we remove the third column of $K$. We then regard the expression in (12) as a $(3 n-2)$ by four system of linear equations. In this case, $n=2$ sensors generates a $(4 \times 4) \mathrm{K}$ matrix so that (12) is a determined system with direct solution $f$ $=\mathbf{K}^{1} \mathbf{g}$. Hence, the LP method can be used by an experimenter that has only two sensors, each measuring bearing, arrival time, and field amplitude. In this case, source location $(x, y)$, time of occurrence $t$, and source strength $s$ can be retrieved. If the two sensors do not provide field amplitude information, the experimenter can still retrieve the flash location and time of occurrence; that is, $(12)$ becomes a $(3 \times 3)$ system of linear equations, and $\mathbf{f}=\operatorname{col}\left(x, y, d_{i}\right)$.

If $\delta$ is unity, the row vectors of $\mathbf{K}$ involving $\sin \phi$. and $\cos \phi$, appear numerically small, that is, like a zero vector, relative to the other row vectors of $\mathbf{K}$, and the matrix is ill-conditioned for many source locations when only three ALDF sensors are available.

To avoid unstable inversions associated with an illconditioned $\mathbf{K}$-matrix, we have made the assignment $\delta$ $=10^{3} \mathrm{~m}$. This increases the magnitude of the small trigonometric components of $\mathbf{K}$ and effectively filters small eigenvalues; see section $3 \mathrm{~d}$ below and appendix $A$ for additional details regarding the value of $\delta$. Other, more sophisticated means of filtering small eigenvalues by adding external physical constraints to the solution process are discussed in Twomey (1977, Chapter 6).

\section{Simulated tests of the LP method}

\section{a. Overview}

Because the effects of propagation are known to degrade the quality of field amplitude data, $F$. [Cooray 1987, his Eq. (2)], and because we have not taken specific measures to model and correct for these errors, we will not consider these data in this and all tests to follow. We also assume that all sources and sensors are located on the surface of a spherical earth. By selecting a known source lat-long location, we generate the true arrival times and bearings to each sensor. Simulated measurements are generated by adding errors to the computed arrival times and bearings. The errors are chosen from a uniform random distribution. Although the bulk magnitude of errors that we will choose are typical of a real experiment, we have not attempted to simulate the detailed effects of propagation error. Such effects (with regard to arrival time retrievals) are provided by Honma et al. (1998).

Next, the simulated measurements are analyzed with the LP method. Since the LP method is a planar model, we must establish a convention for mapping source and sensor locations (expressed in degrees of latitude and longitude) to locations in the $x-y$ plane of a standard Cartesian coordinate system. We then apply the LP method to solve the problem in the Cartesian system. Next, an inverse mapping is used to convert the $(x, y)$ solution back into latitude and longitude coordinates on the surface of the earth. At this point, the lat-long solution can be compared with the known source to assess true location error.

If one assumes a flat earth and performs the entire simulation within a Cartesian coordinate system, the resulting retrieval errors are smaller. This is because one avoids errors due to earth curvature and the numerical errors associated with spherical/Cartesian system mappings. Because in any real field experiment the source retrievals are ultimately referenced to the spherical earth, we include the net effects of earth sphericity in this and all other simulations provided below. 


\section{b. Spherical arrival time and bearing}

Figure 2 indicates how to compute the arrival time $t_{i}$ and bearing $\phi_{i}$ for the $i$ th sensor on a sphere. The unit vectors pointing from the origin $\mathrm{O}$ to the $i$ th sensor $(M)$, to the lightning source $(L)$, and to the North Pole $(N)$ are, respectively,

$$
\begin{aligned}
\hat{\mathbf{r}}_{i} & =\cos \varphi_{i} \cos \lambda_{i} \hat{\mathbf{u}}+\cos \varphi_{i} \sin \lambda_{i} \hat{\mathbf{v}}+\sin \varphi_{i} \hat{\mathbf{w}} \\
\hat{\mathbf{r}} & =\cos \varphi \cos \lambda \hat{\mathbf{u}}+\cos \varphi \sin \lambda \hat{\mathbf{v}}+\sin \varphi \hat{\mathbf{w}} \\
\hat{\mathbf{r}}_{N} & =\hat{\mathbf{w}}
\end{aligned}
$$

Using the law of cosines from spherical trigonometry gives the spherical angle $A_{i}$,

$$
A_{i}=\frac{180}{\pi} \cos ^{-1}\left(\frac{\cos a-\cos b_{i} \cos c_{i}}{\sin b_{i} \sin c_{i}}\right)
$$

where $a=\cos ^{-1}\left(\hat{\mathbf{r}}_{N} \cdot \hat{\mathbf{r}}\right), b_{i}=\cos { }^{1}\left(\hat{\mathbf{r}}_{N} \cdot \hat{\mathbf{r}}_{i}\right), c_{i}=$ $\cos ^{-1}\left(\hat{\mathbf{r}}_{i} \cdot \hat{\mathbf{r}}\right)=(1 / R) c t_{i}, R=$ radius of earth, and by convention the lightning source activates at $t \equiv 0$. The angle, $A_{i}$, varies between $0^{\circ}$ (north) and $180^{\circ}$ (south). We correct $A$, to construct the bearing function $\phi_{\text {, that }}$ varies in the following manner: $0^{\circ}$ (east), $90^{\circ}$ (north), $180^{\circ}$ (west), $270^{\circ}$ (south); that is,

$\phi_{i}=90-A_{i} \quad$ (northeast sources),

$\phi_{i}=90+A_{i} \quad$ (northwest and southwest sources),

$\phi_{i}=450-A_{i} \quad$ (southeast sources).

\section{c. Mappings}

In general, different mappings produce different retrieval errors. We consider two possible approaches: Mapping \#1 (chosen for its mathematical simplicity), and Mapping \#2 (chosen for its orthogonality). In Mapping \#1, we have

$$
x=\left(\lambda-\lambda_{1}\right) R \cos \varphi_{1} \quad y=\left(\varphi-\varphi_{1}\right) R,
$$

where $(\lambda, \varphi)$ is an arbitrary longitude and latitude, respectively. The origin of the Cartesian coordinate system has been arbitrarily selected as site $i=1$; that is, the ordered pair $\left(\lambda_{1}, \varphi_{1}\right)$ is the location of site 1 and $x(\lambda$ $\left.=\lambda_{1}, \varphi=\varphi_{1}\right)=0, y\left(\lambda=\lambda_{1}, \varphi=\varphi_{1}\right)=0$. Note that $y$ is measured along a great circle, that is, a longitude belt, but that $x$ is measured along a latitude belt [which is only a great circle if $\varphi_{1}=0$ (the equator)].

In the second approach, or Mapping \#2, we insist that both $x$ and $y$ are measured along great circles. To do this, we consider an orthogonal system $(\hat{\mathbf{u}}, \hat{\mathbf{v}}, \hat{\mathbf{w}})$ where $\hat{\mathbf{u}}$ is a unit vector directed from the center of the earth to the intersection of the prime meridian and equator, $\hat{\mathbf{w}}$ is a unit vector directed from the center of the earth to the North Pole, and $\hat{v}$ completes the ordered triple in accordance with the right-hand rule, that is, $\hat{\mathbf{v}} \equiv \hat{\mathbf{w}} \times$

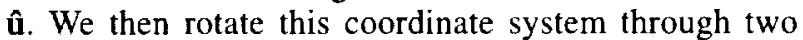
Euler angles $\left(\lambda_{1}, \varphi_{1}\right)$ and define the new resultant ("starred") system as $\left(\hat{\mathbf{u}}^{*}, \hat{\mathbf{v}}^{*}, \hat{\mathbf{w}}^{*}\right)$. In the starred system, $\hat{\mathbf{u}}^{*}$ is directed from the center of the earth to site 1. Mapping \#2 is then

$$
x=R \lambda^{*}(\lambda, \varphi) \quad y=R \varphi^{*}(\lambda, \varphi),
$$

where

$$
\begin{aligned}
& \lambda^{*}(\lambda, \varphi)=\tan ^{-1}\left(\frac{\cos \varphi \sin \lambda \cos \lambda_{1}-\cos \varphi \cos \lambda \sin \lambda_{1}}{\cos \varphi \cos \lambda \cos \lambda_{1} \cos \varphi_{1}+\cos \varphi \sin \lambda \sin \lambda_{1} \cos \varphi_{1}+\sin \varphi \sin \varphi_{1}}\right) \\
& \varphi^{*}(\lambda, \varphi)=\sin ^{-1}\left(\sin \varphi \cos \varphi_{1}-\cos \varphi \cos \lambda \cos \lambda_{1} \sin \varphi_{1}-\cos \varphi \sin \lambda \sin \lambda_{1} \sin \varphi_{1}\right) .
\end{aligned}
$$

Again, one can verify from (18) and (19) that $x(\lambda=$ $\left.\lambda_{1}, \varphi=\varphi_{1}\right)=0, y\left(\lambda=\lambda_{1}, \varphi=\varphi_{1}\right)=0$. The arctangent expression in (19) must be appropriately corrected depending on what quadrant (northeast, northwest, southeast, southwest) the point $(\lambda, \varphi)$ is relative to site 1 .

\section{d. Simulation}

We first consider three ALDF sites in the Darwin, Australia, region that were used as part of the MCTEX described in Keenan et al. (1994, 1996). Computer-generated lightning sources were spaced $0.02^{\circ}(\sim 2 \mathrm{~km})$ apart across the analysis region. Figure $3 \mathrm{a}$ shows the spatial distribution of the retrieved horizontal source location error, contoured in units of kilometers, when no experimental errors are considered and when Mapping \#1 is used. The retrieved location errors are within $1 \mathrm{~km}$ for regions inside the ALDF network.

Since no experimental errors have been added to the simulated values of the arrival times and bearings, the retrieval errors shown in Fig. 3a are due solely to earth curvature and numerical truncation error. We originally performed these simulations, and all simulations to follow, assuming a flat earth. When this was done, all of our methods gave retrieval errors well below $2.5 \mathrm{~m}$ across the entire analysis region when no measurement errors were involved. This is to be expected since our 


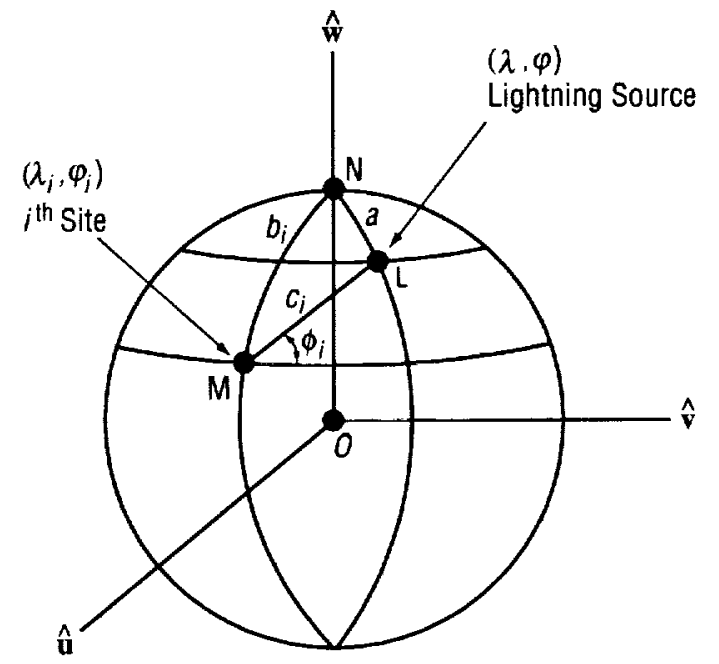

FiG. 2. Spherical trigonometry used for determining anival time and bearing.

methods are exact solutions for the plane (the $2.5-\mathrm{m}$ error maximum occurred only in the LP method over a limited portion of the analysis region and was an artifact of what accuracy level we required of our iterative matrix inversion routine). Hence, compared to the negligible errors obtained from the flat earth simulations, the errors shown in Fig. 3a are effectively due to earth curvature alone. However, the amount of retrieval error due to earth curvature depends on what cartesian-to-spherical coordinate system mapping is used (e.g., Mapping \#1 or Mapping \#2). Because one will always be interested in how much retrieval error the planar models acquire due to earth curvature, all simulations below show retrievals first without added measurement errors, as in Fig. 3a.

When experimental errors are included in the simulation, we obtain the retrieved location errors given in Fig. 3b. The retrieved errors are mean values obtained from performing 100 individual retrievals at each trial location. For each of the 100 trials, an arrival time error selected from a uniform random distribution (ranging from -300 to $300 \mathrm{~ns}$ ) is added to the arrival time value, and a bearing error (ranging from $-2^{\circ}$ to $2^{\circ}$ ) is added to the bearing value. In addition, we have simulated sensor location errors by purposely entering into the LP method false site locations (with an error as great as $1 / 2$ $m)$; the sensor location errors have remained fixed for all source analyses. As expected, the addition of experimental errors increases location retrieval errors, but the retrieved errors are still within $10 \mathrm{~km}$ for a large portion of the analysis region. Roughly speaking (that is, not accounting for earth curvature errors, truncation errors, or other errors due to matrix inversion), a 300ns timing error multiplied by the speed of light gives only a $90-\mathrm{m}$ error, and a $2^{\circ}$ error at a range of $300 \mathrm{~km}$ is about $10 \mathrm{~km}$.

When Mapping \#2 is used instead of Mapping \#1, (a)
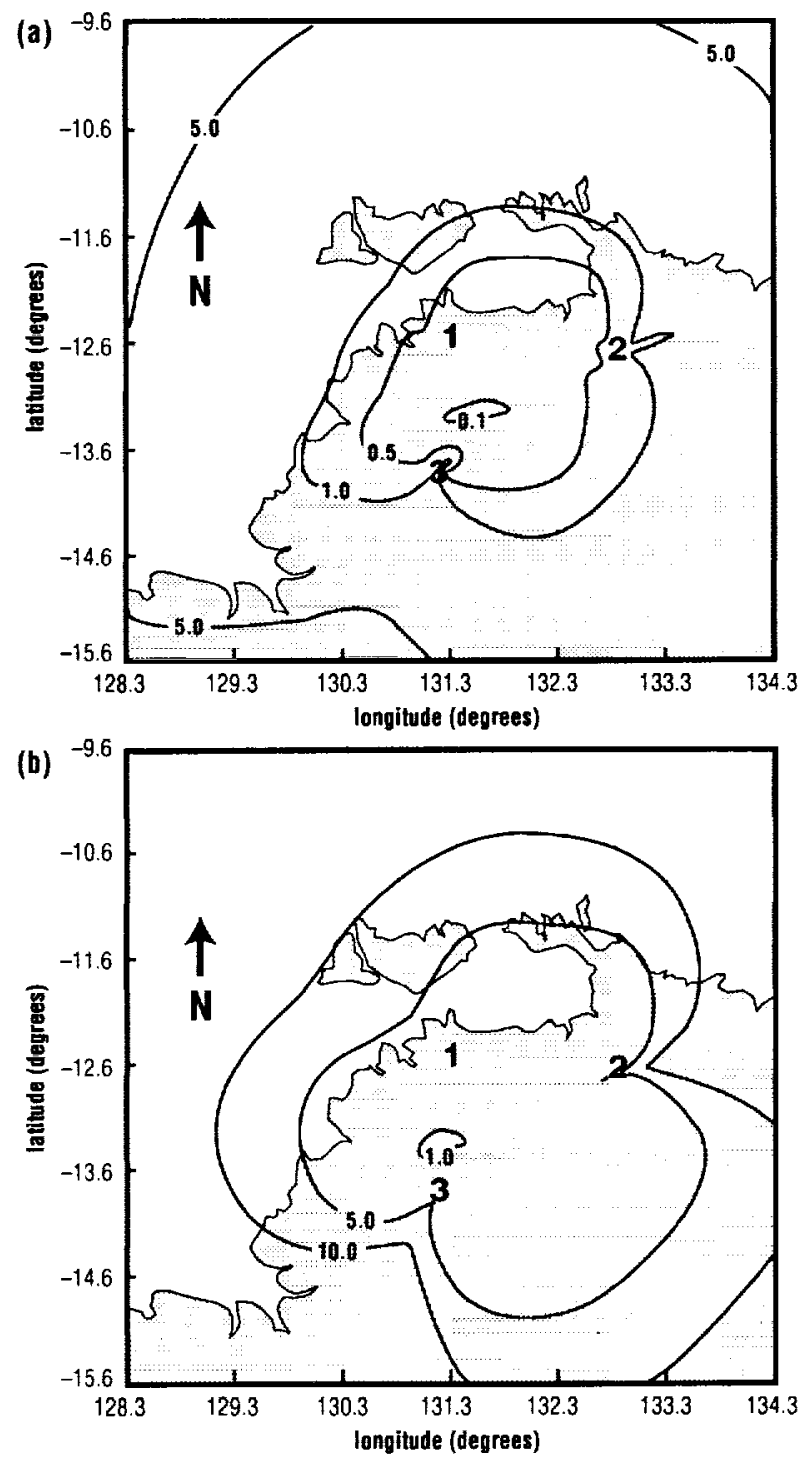

FIG. 3. Lightning location retrieval errors for a three-station network using the LP method: (a) no measurement errors, and (b) with the following random measurement errors: 0.5 -m sensor location error, 300 -ns timing error, $2^{\circ}$ bearing error. Mapping $\# 1$ is used. Contours are in units of kilometers. The analysis region shown is $6^{\circ}$ lat $(667 \mathrm{~km}) \times 6^{\circ}$ long (about $\left.651 \mathrm{~km}\right)$, and this is where the Maritime Continent Thunderstorm Experiment (MCTEX) was conducted.

we obtain the results shown in Figs. 4a,b. As before, no experimental errors have been added to the sensor positions, arrival times, and bearings in the results of Fig. 4a, but the results in Fig. $4 \mathrm{~b}$ include these errors. The results in Fig. 4a appear somewhat better than those in Fig. 3a, but the results in Figs. $3 \mathrm{~b}$ and $4 \mathrm{~b}$ are similar since the simulated experimental errors tend to mask differences between Mapping \#1 and Mapping \#2.

Figures $5 \mathrm{a}-\mathrm{b}$ and $6 \mathrm{a}-\mathrm{b}$ show all of the same type of analyses just described, but for the case of four ALDF sensors. The additional sensor clearly helps reduce re- 

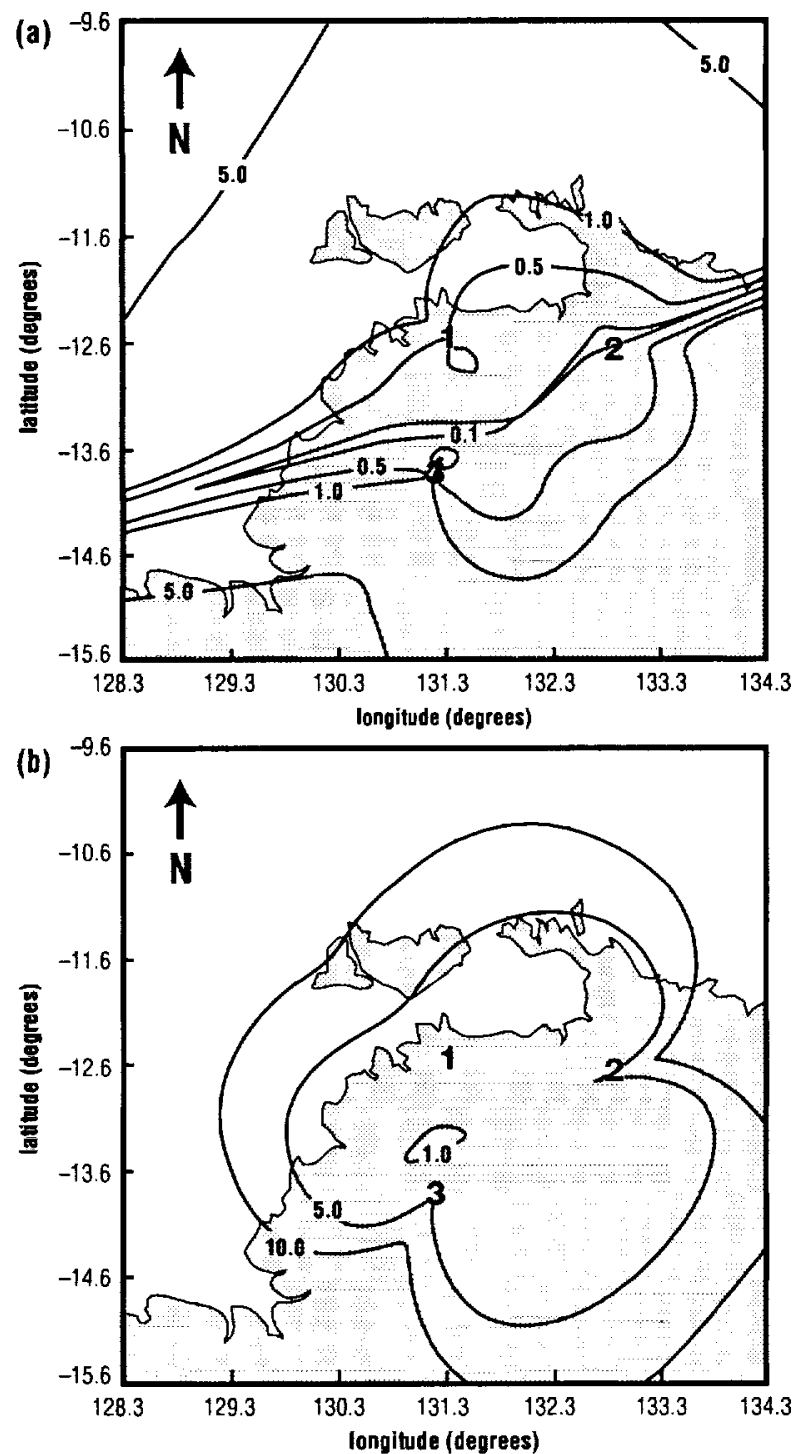

FIC. 4. Same as in Fig. 3 except that Mapping $\# 2$ is used.

trieval error. In addition, Mapping \#2 produces smaller retrieval errors than Mapping \#1.

In summary, our simulations show that there are two basic principles operating here: 1) Mapping \#2 generally outperforms Mapping \#1, and 2) four-station networks are more resilient to measurement errors than threestation networks. Principle 1 is supported by the following: (i) the error in Fig. $4 \mathrm{a}$ is less than in Fig. 3a, and (ii) the error in Fig. 6a is less than in Fig. 5a. Support for principle 2 is also evident. For the three-station network there are large increases in error going from Fig. $3 a$ to Fig. 3b and from Fig. 4a to Fig. 4b, but for the four-station network there is not as much increase in error (see error changes going from Fig. 5a to Fig. 5b, and from Fig. 6a to Fig. 6b).

Next, a word regarding bearing data. When four sensors are available, bearing data are not needed to obtain
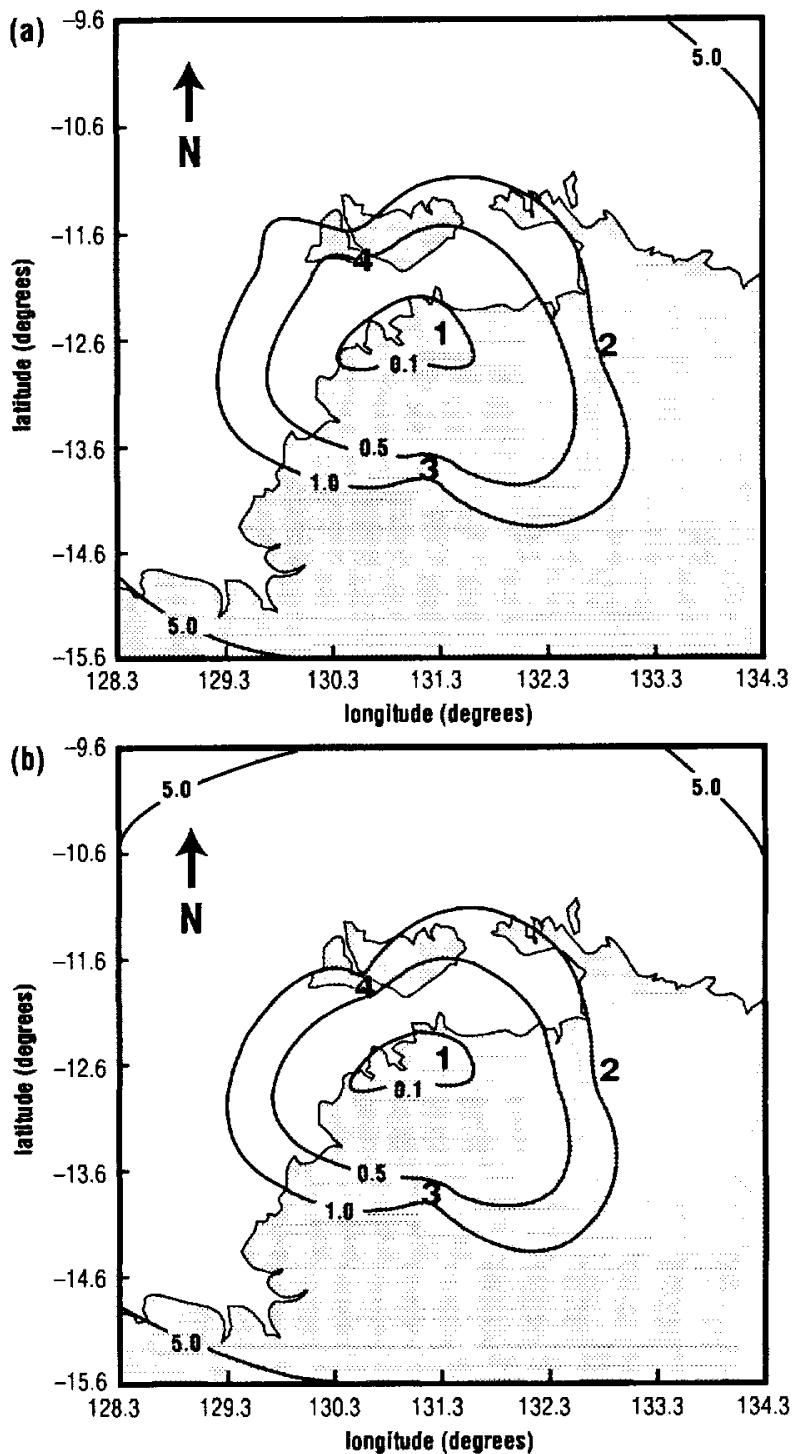

FiG. 5. Same as in Fig. 3 except that four sensors are used.

lightning location retrievals [see (11)]. By removing the bearing data from the four-station MCTEX region simulations, that is assigning $\delta=0$, and by applying Mapping \#2 we obtained virtually the same results as those presented in Figs. $6 \mathrm{a}, \mathrm{b}$. This is because the weighting factor $\delta$ introduced into (11) to generate Figs. $6 a, b$ is relatively small (i.e., $10^{3}$ ) so that bearing data has little influence on the final solution. We also find little change in the solutions for intermediate values, $\delta=10,10^{2}$. However, as we increase $\delta$ from $10^{3}$ to $10^{4}, 10^{5}$, and $10^{6}$, the retrieval errors increase (see appendix A for more details).

For a three-sensor ALDF network, bearing data plays a more profound role in helping to constrain the solution space. If $\delta=0$ (no bearing data used), there would be fewer constraint equations than unknowns, and one would not be able to obtain a solution using the LP 

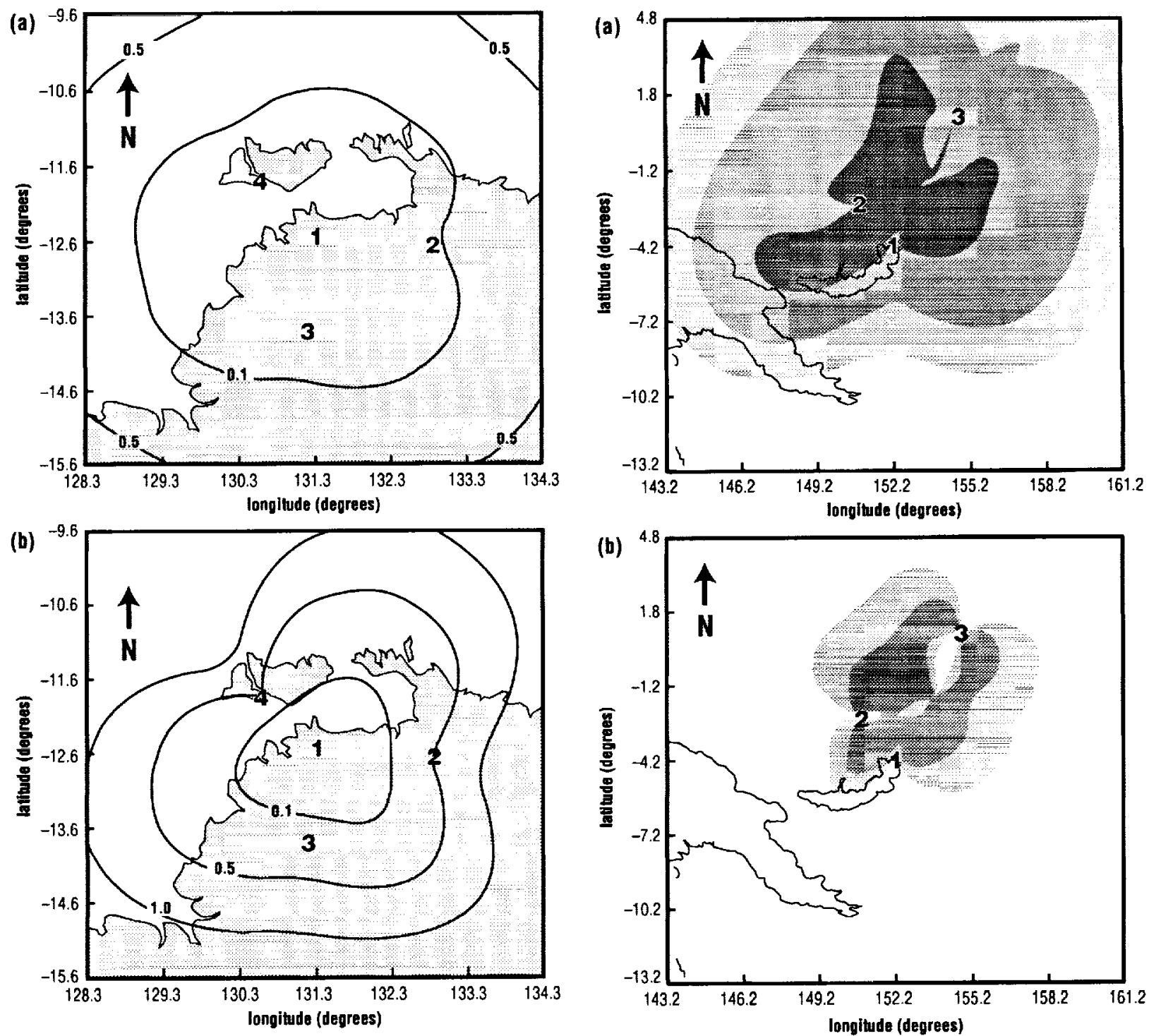

FIG. 6. Same as in Fig. 3 except that four sensors and Mapping \#2 are used.

formalism. (Note: a different formalism to be described in section 4 below can be used to find solutions over a substantial portion of the analysis region using just three arrival time sensors.) As noted above, when $\delta=1$ the $\mathrm{K}$ matrix is ill-conditioned for many source locations, leading to poor retrieval results. When an iterative method is used to invert an ill-conditioned K-matrix, the computer time will be excessive. If a standard analytic form for $\mathbf{K}$ ' is used (this is practical since the dimensions of $\mathbf{K}$ are small), then the time required to obtain the inverse is comparatively short and does not change. However, in either case, error magnification is excessive. When $\delta=10,10^{2}, 10^{3}$, or $10^{4}$, there is no appreciable change in retrieval error and error magnification is minimal. When $\delta=10^{5}$, the retrieval errors begin to increase slightly because of bearing errors.

Three-station LP simulations for different values of $\delta$ are also provided in appendix $\mathrm{A}$.

For comparison, we also provide error results (Figs. $7 \mathrm{a}, \mathrm{b})$ for the three sites used in the Tropical Ocean and Global Atmosphere Coupled Ocean-Atmosphere Response Experiment (TOGA COARE) described in Peterson et al. (1996) and Orville et al. (1997). This experiment employed a larger sensor baseline than that

FIG. 7. Same as in Fig. 3 except that this is for the TOGA COARE analysis region, and Mapping $\# 2$ is used. The analysis region is $18^{\circ}$ lat $(2002 \mathrm{~km}) \times 18^{\circ}$ long (about $\left.1996 \mathrm{~km}\right)$. Shading, rather than contouring, is used to clarify the nonmonotonic distribution of retrieval errors. 
used in MCTEX, and our simulated tests cover an analysis region $18^{\circ} \times 18^{\circ}$ in latitude and longitude. The sources in this simulation where placed $0.05^{\circ}$ apart and Mapping \#2 was used; as with the three-sensor MCTEX study, the retrieval errors for Mapping \#2 differ little from those errors obtained using Mapping \#1. Overall, the $2^{\circ}$ bearing error and the effects of earth curvature make it difficult to obtain errors below $10 \mathrm{~km}$ for distant sources. In addition, the TOGA COARE network geometry is not optimum since the baseline between sites 1 and 2 is not very large in comparison to the other two baseline distances.

\section{Quadratic Planar (QP) method}

In this section we assume that only three arrival time measurements are available from the ALDF network. Hence, the methods of section 2 cannot be applied, but some insight about the source location can still be obtained. We assume that sensor $i=1$ is at the origin of a rectangular Cartesian coordinate system (i.e., $x_{1}=y_{1}$ $=z_{1}=0$ ), we specify the convention $t_{1} \equiv 0$, and we again take $z=0$. This leads to one nonlinear equation and two linear equations all in the three unknowns $(x, y, r)$ :

$$
\begin{array}{rlrl}
x^{2}+y^{2} & =r^{2} ; & & i=1 \\
q_{i}^{\prime} & \equiv \frac{1}{2}\left(r_{i}^{2}-c^{2} t_{i}^{2}\right) & & \\
& =x_{i} x+y_{i} y+c t_{i} r ; & i=2,3 .
\end{array}
$$

The equations in (20) were derived from the transit equation in (1) by means similar to that discussed prior to (2) of section 2. We have removed the source activation time $t$ with the relation $t=-r / c$. Since $t_{\mathrm{i}} \equiv 0$, it is consistent that $t \leq 0$ given that $r \geq 0$. Each sensor is a distance $r_{i}=\left(x_{i}^{2}+y_{i}^{2}\right)^{1 / 2}$ from the origin.

With minimal algebra, it can be shown that each equation in (20) can be transformed into the equation of a circle with radius $c\left(t_{1}-t\right)$ and center at $\left(x_{i}, y_{i}\right)$, where $i=1,2,3$. We will see below that the source is located where the three circles intersect. We will also find that certain source locations produce arrival time data that can be described geometrically by two possible sets of three circles. Each set of three circles define a unique intersection point in the $x-y$ plane, thereby leading to a fundamental ambiguity in source location retrieval.

Geometric intersections of the circular curves described above are obtained by solving the system of equations in (20). To solve the system, we first subtract the terms $c t, r$ from each side of the linear equation set:

$$
q_{i, 1} \equiv q_{i}^{\prime}-c t_{1} r=x_{i} x+y_{i} y ; \quad i=2,3 .
$$

Identifying the vectors, $\mathbf{q}=\operatorname{col}\left(q_{1}, q_{2}\right), \mathbf{r}=\operatorname{col}(x, y)$, we may write

$$
\mathbf{q}=\mathbf{Q} \mathbf{r}
$$

where the $\mathbf{Q}$-matrix and its inverse are given by

$$
\mathbf{Q}=\left[\begin{array}{ll}
x_{2} & y_{2} \\
x_{3} & y_{3}
\end{array}\right], \quad \mathbf{Q}^{1}=\frac{1}{x_{2} y_{3}-y_{2} x_{3}}\left[\begin{array}{cc}
y_{3} & -y_{2} \\
-x_{3} & x_{2}
\end{array}\right] .
$$

From (22) and (23) and our discussion preceding (21) we have the relations

$$
\begin{aligned}
x(r) & =\left(y_{3} q_{1}-y_{2} q_{2}\right) /\left(x_{2} y_{3}-y_{2} x_{3}\right) \\
y(r) & =\left(x_{2} q_{2}-x_{3} q_{1}\right) /\left(x_{2} y_{3}-y_{2} x_{3}\right) \\
t(r) & =-r / c .
\end{aligned}
$$

The $x$ and $y$ variables are written as functions of $r$ in (24) since the components of $q$ depend on $r$ as given in (21). Substituting the first two equations of (24) into the first (nonlinear) equation of (20) and carrying out the algebra leads to an equation quadratic in $r$ alone:

$$
A r^{2}+B r+C=0
$$

where

$$
\begin{aligned}
& A=c^{2}\left[r_{3}^{2} t_{2}^{2}-2\left(x_{2} x_{3}+y_{2} y_{3}\right) t_{2} t_{3}+r_{2}^{2} t_{3}^{2}\right]-\left(x_{2} y_{3}-y_{2} x_{3}\right)^{2} \\
& B=2 c\left[-r_{3}^{2} q_{2}^{\prime} t_{2}+\left(x_{2} x_{3}+y_{2} y_{3}\right)\left(q_{2}^{\prime} t_{3}+q_{3}^{\prime} t_{2}\right)-r_{2}^{2} q_{3}^{\prime} t_{3}\right] \\
& C=r_{3}^{2} q_{2}^{\prime 2}-2\left(x_{2} x_{3}+y_{2} y_{3}\right) q_{2}^{\prime} q_{3}^{\prime}+r_{2}^{2} q_{3}^{\prime 2} .
\end{aligned}
$$

Hence, the lightning source range, $r$, is the nonnegative real root obtained from the formal (two root) solution:

$$
r=\left\{\begin{array}{l}
r_{+}=\frac{-B+\sqrt{B^{2}-4 A C}}{2 A} \\
r_{-}=\frac{-B-\sqrt{B^{2}-4 A C}}{2 A} .
\end{array}\right.
$$

Values of $r=0$ correspond to a direct lightning strike of sensor $i=1$, which we ignore. Note from (26) that the numerical value of the coefficients $(A, B, C)$ are obtained from the sensor locations and excitation times, that is, on the six variables: $\left\{x_{2}, y_{2}, t_{2}, x_{3}, y_{3}, t_{3}\right\}$; the variables, $q_{i}^{\prime}$, are obtained from the expressions $\frac{1}{2}\left(r_{i}^{2}-\right.$ $\left.c^{2} t^{2}\right)$ as given in the last two equations of (20). After these data are used to compute $r,(24)$ is used to find the lightning location $[x(r), y(r)]$, and time of occurrence, $t(r)$.

\section{Simulated tests of the QP method}

By placing computer-generated lightning sources $0.02^{\circ}$ apart in latitude and longitude across the analysis area (see section 3), we have determined the horizontal location error resulting from each root in (27). To facilitate comparisons with simulated tests of the LP method, sensor position and arrival time errors used here are as described in section $3 \mathrm{~d}$ and 100 trials at each source location are once again used to generate mean retrieval location errors. Mapping \#I given in (17) is employed.

Figure 8 clarifies what root provides the correct source location. The shaded regions are where $r_{+}$is correct and the unshaded region is where $r_{-}$is correct. 
Interestingly, the dividing lines of these regions are defined by the sensor baselines.

When we pick only the correct root and plot the associated error result over the analysis region, we obtain the result given in Fig. 9a. When a 300-ns uniform random error is added to the computer-generated arrival times, we obtain the mean horizontal distance errors given in Fig. 9b. Considering that only three sensors are involved, retrieval errors are quite good; a large region of errors below $1 \mathrm{~km}$ is evident. Distant sources, or sources located near the outer sensor baselines are more difficult to accurately retrieve. See appendix B for a definition of outer sensor baseline. A comparison between the three-station LP results in Fig. $3 \mathrm{~b}$ shows that the QP method provides better results over most of the analysis region. This is due in part to the large $2^{\circ}$ bearing errors implemented in the LP simulation and the fact that the three-station LP method depends on bearing data to obtain a solution. The four-station LP method (Fig. 5b) outperforms the QP method in most locations.

Since bearing data can aid in determining which root, $r_{+}$or $r_{-}$, is correct (see section 6a below on solution ambiguity) and since the QP method generally gives better results than the three-station LP method, it is evidently better to use the QP method than the threestation LP method when bearing data are available (provided the source is not located near an outer sensor baseline). This conclusion is based, of course, on an assumed bearing error of $2^{\circ}$.

\section{Examination of $Q P$ method roots}

When applying the QP method to actual arrival time data, one picks the solution associated with a nonnegative real root, that is, the source range $r$ must be nonnegative and real. A detailed discussion of root results is provided below.

\section{a. Unequal nonnegative real roots (ambiguities)}

In the discussion of three sensor networks by Holle and Lopez $(1993$, pp. 8, 11), the lightning source location $(x, y)$ is described in terms of the intersection of two hyperbola branches; each branch is defined by two sensors. For some lightning source locations, the hyperbola branches intersect at two locations [see, for instance, Fig. 6, p. 11 of Holle and Lopez (1993)]. This amounts to a fundamental ambiguity in location retrieval and the authors correctly assert that the ambiguity can be removed by adding a fourth (properly positioned) sensor. Ambiguities are described in our formalism by the intersection of circles as indicated in section 4. To fully appreciate the hyperbolic and circular geometrical viewpoints, it is important to recognize that the two intersection points defined by two sets of three circles are identical to the double intersections obtained from the two hyperbola branches mentioned above. In other

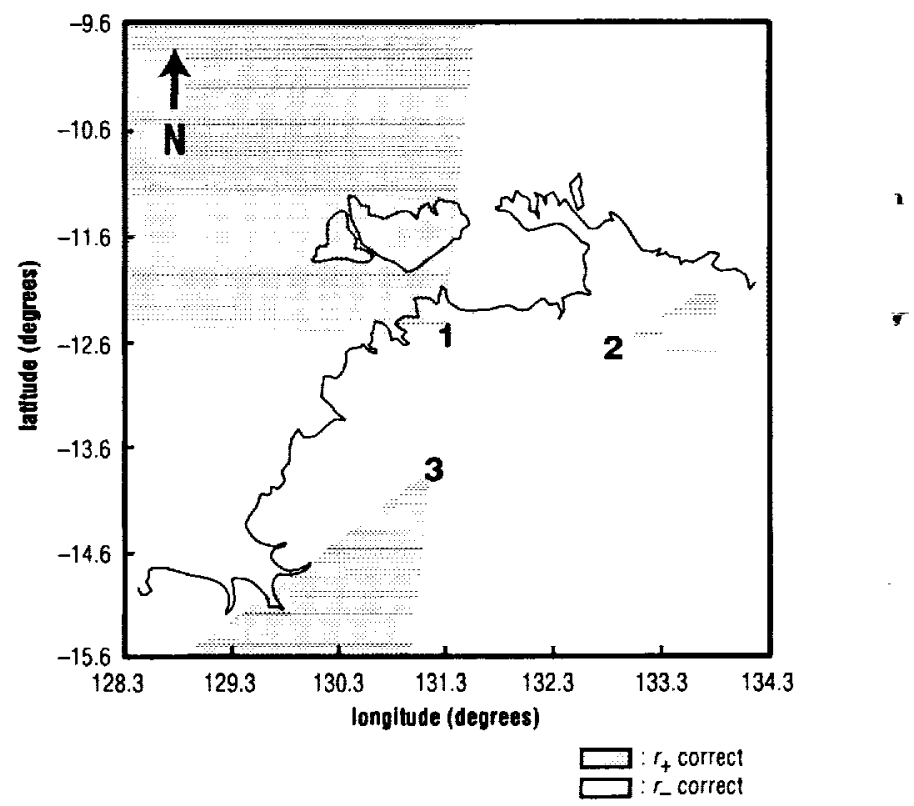

FIG. 8. Comparison of roots in the QP method. The shaded regions indicate where root $r_{+}$is the correct solution and the unshaded region indicate where root $r_{-}$is the correct solution. No measurement crrors have been added to the simulated data.

words, these two widely different geometrical viewpoints produce identical results, as they must.

Without reference to the geometry of hyperbolic or circular intersections, our algebraic formalism immediately defines all ambiguous cases. An ambiguity will exist whenever two unequal nonnegative real roots result from (27). In order to determine what lightning source locations produce these "ambiguity regions," we have kept a record of the root results in the numerical experiments described in section 5 above. For the case of no simulated experimental errors, the source locations that resulted in two unequal nonnegative real roots are indicated by the shaded regions in Fig. 10; see section $6 \mathrm{~b}$ below for minor corrections to the ambiguity regions. In general, a different network geometry would produce different results.

Strictly speaking, since two distinct sources can produce identical arrival time difference information, there is no means of discriminating which source location is correct unless some additional information is supplied to the retrieval process. In effect, the solution is fundamentally nonunique. [Similar comments about the retrieval of charge from ground-based field measurements have been made in Koshak and Krider (1994). In that problem, a point charge $Q_{v}$ and a sphere of radius $a$ with total charge $Q_{\text {. }}$ produce identical electrostatic fields outside the radius $a$.] Hence, additional measurements (e.g., arrival time, bearing, signal amplitude, radar, acoustical, interferometric) must be used to pick the correct root. Bearing data would be the most common data to use in root discrimination since it is part of the ALDF data stream. 

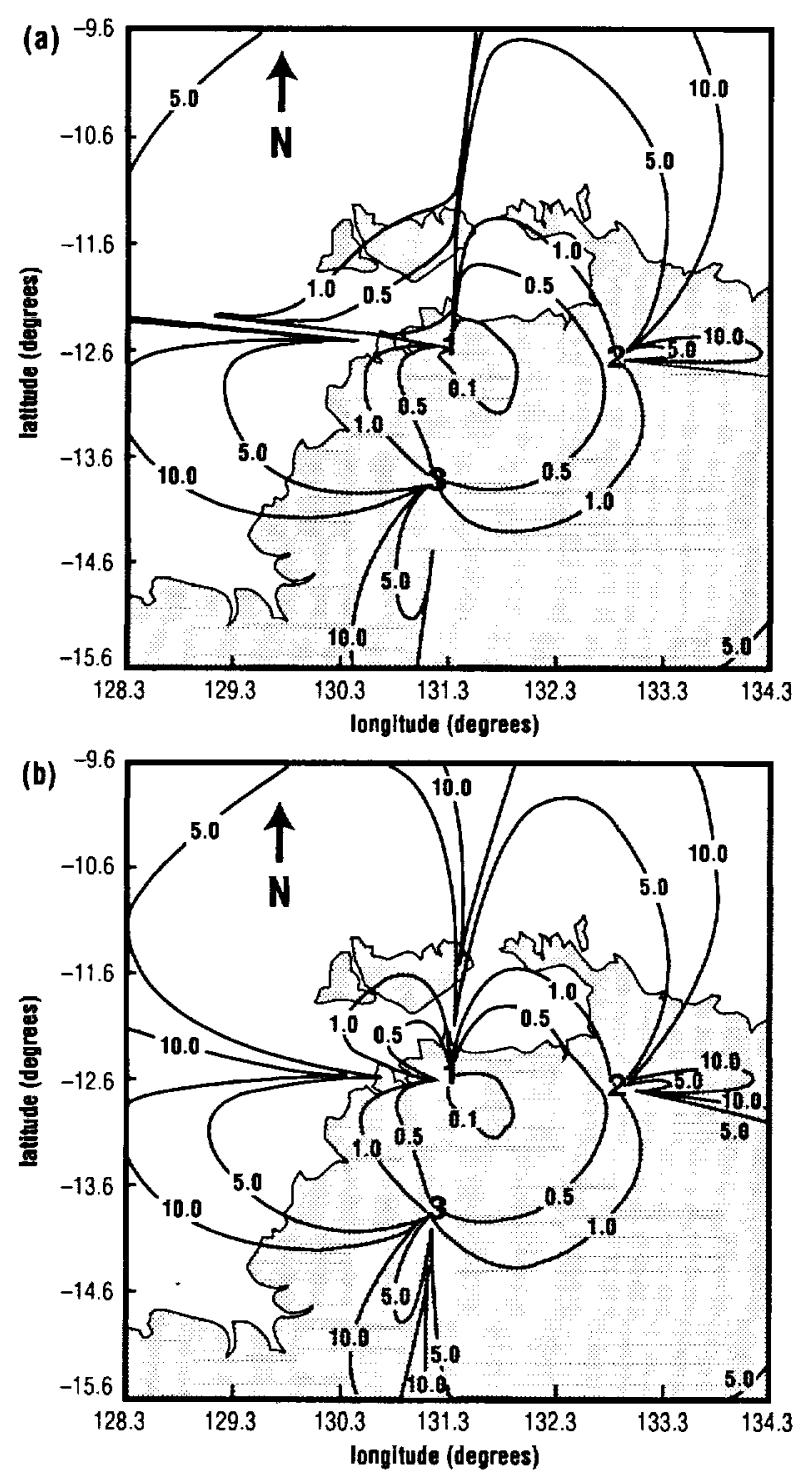

F1G. 9. Retrieval errors from the correct root in the QP method: (a) no measurement errors, and (b) with the following random measurement errors: 0.5-m sensor location error, 300-ns timing error. Mapping \#I is used. Contours in kilometers.

Nonetheless, an experimenter might be tempted to compare the shaded regions in Figs, 8 and 10 in order to determine which of the two unequal nonnegative real roots produce the true source location. However, one must remember that Fig. 8 does not pose a real physical constraint to an unknown source since it only provides information if the source location is already known; obviously in a real field experiment the source location is not yet known.

Additional rigor clarifies the immutability of the ambiguous case. Note that there are three ambiguity regions in Fig. 10 , and call the regions $\left\{R_{1}, R_{2}, R_{3}\right\}$. Similarly, there are three regions $\left\{P_{1}, P_{2}, P_{3}\right\}$ in Fig. 8 that are wholly contained within each of the respective ambi-

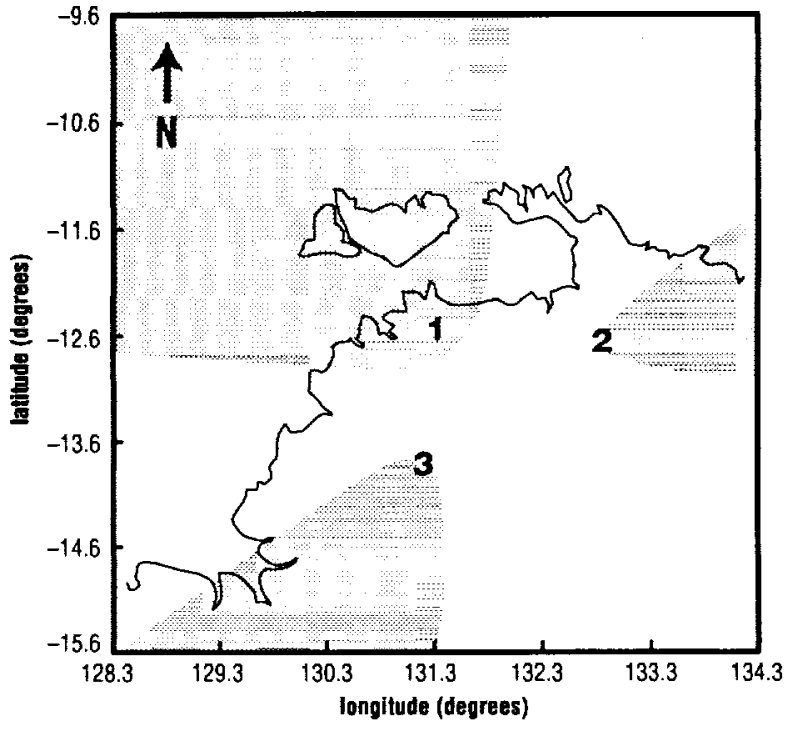

Fici. 10. Shaded regions are the QP method "ambiguity regions" that indicate what lightning source locations result in two unequal nonnegative real roots. No errors were added to the simulated arrival times.

guity regions. If one subtracts the respective regions $\left\{P_{1}, P_{2}, P_{3}\right\}$ from the respective regions $\left\{R_{1}, R_{2}, R_{3}\right\}$, one obtains the three regions $\left\{N_{1}, N_{2}, N_{3}\right\} \equiv\left\{R_{1}-\right.$ $\left.P_{1}, R_{2}-P_{2}, R_{3}-P_{3}\right\}$. When two unequal nonnegative real roots are obtained, we find that $r_{+}$occurs in $P$, and $r_{-}$occurs in $N_{i}$, that is, the pair of roots produce solutions in $\left(P_{1}, N_{1}\right),\left(P_{2}, N_{2}\right)$, or $\left(P_{3}, N_{3}\right)$. From Fig. 8, region $P_{i}$ is the region where $r_{+}$is correct, $N_{1}$ is the region where $r_{-}$is correct, and both $P_{\text {, }}$ and $N$, are subregions of $R_{t}$. In other words, each solution is possibly correct so that comparisons between Figs. 8 and 10 serve no help in determining the correct root.

Nonetheless, $r_{+}$is correct for most of the ambiguity region shown in Fig. 10. Hence, a MCTEX experimenter who obtains two nonnegative real roots, but does not have ancillary datasets such as radar, magnetic bearing, etc. (for determining the correct root) is best off selecting the root $r_{+}$.

\section{b. Equal nonnegative real roots}

In this section we are interested in identifying what source locations produce two equal nonnegative real roots. Note that this condition is satisfied when the discriminant, $B^{2}-4 A C$, in (27) is zero, that is, the two equivalent roots correspond to a unique (unambiguous) solution $[x(r), y(r), t(r)]$ where $r_{+}=r_{-}=r$. In appendix $B$, we show for arbitrary noncollinear network geometries that the discriminant function is zero only along the outer sensor baselines. Therefore, the ambiguity regions shown in Fig. 10 are technically not ambiguous along these linear domains. 


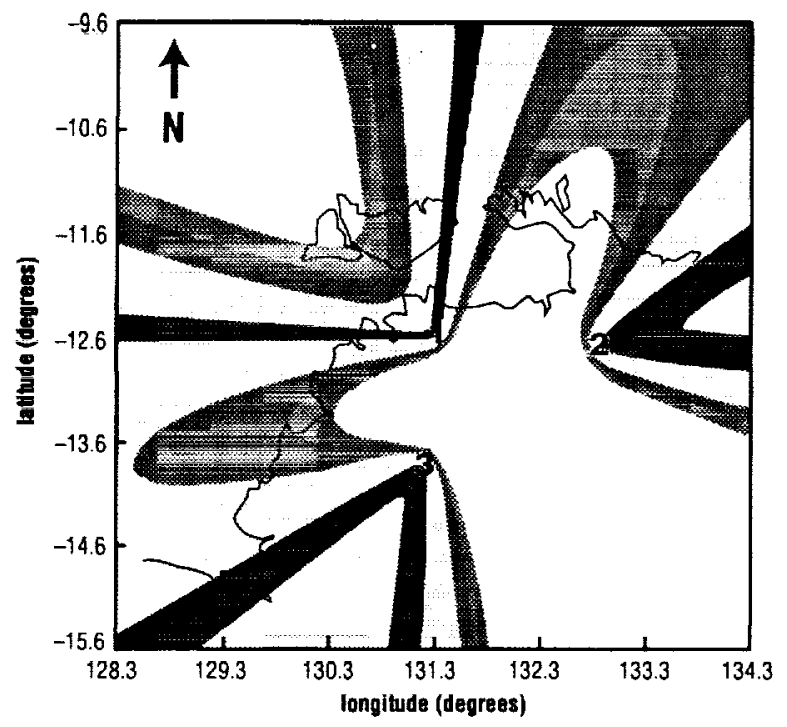

Key: Scaled Value

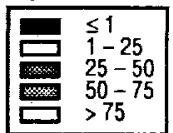

FIG. 11. Plot of the QP method discriminant (scaled by a large arbitrary constant for plotting purposes). Expressions for $A, B$, and $C$ in the discriminant, $B^{2}-4 A C$, are given in (26).

\section{c. Complex roots}

Complex roots occur whenever the discriminant in (27) becomes negative. Figure 11 shows how the discriminant varies for different source locations across the analysis region. As we have already shown in section $6 \mathrm{~b}$ and appendix $\mathrm{B}$, the discriminant is zero for sources located along the outer sensor baselines. Figure 11 shows additionally that the discriminant is a relative minimum at the outer sensor baselines.

From the simulation in section 5 (with 300 -ns arrival time errors, and 100 trials per test location) we have tallied the fraction of trials at each location that produce complex roots. Figure 12 shows that there are no complex roots over most of the analysis region except when the sources are near the outer sensor baselines. These regions (or "spokes") appear to diverge with range from the sensors and as many as $40 \%-60 \%$ of the sources are complex within the spokes. Clearly, for sources located sufficiently close to the outer sensor baselines, measurement errors are occasionally large enough to drive the discriminant negative. Whenever the discriminant is negative, both roots in (27) are complex and no physical solution is obtained. Conversely, whenever complex roots are obtained from a set of actual measurements the source is likely to be located in one of the spoked regions.

\section{d. Overview of root results}

From our discussion so far, we can conclude that any retrieval will produce one of the following cases: (a) $r_{r}$

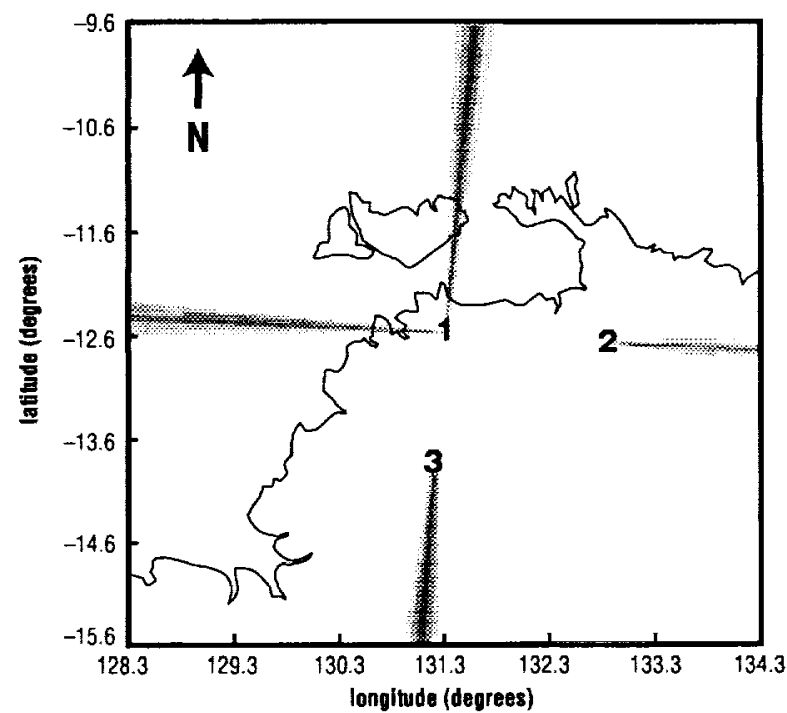

Key: Percenl Complex

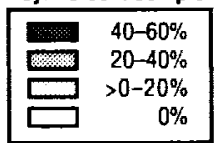

FIG. 12. Fraction of 100 simulated sources at cach location that produce complex roots using the QP method. A $0.5-\mathrm{m}$ sensor location error, a 300-ns timing crror, and Mapping \#1 was used.

$\geq 0, r_{-} \geq 0, r_{+} \neq r_{-}$; (b) $r_{+} \geq 0, r_{-} \geq 0, r_{+}=r$; (c) $r_{+}<0, r_{-} \geq 0$; or (d) $r_{+}$and $r_{-}$complex. Case a corresponds to a source that is located inside one of the ambiguity regions, case b corresponds to a source located on an outer sensor baseline, case corresponds to a source that is not located in any of the ambiguity regions or along any outer sensor baseline, and case d corresponds to a source located on or near any outer sensor baseline when measurement errors are sufficient to drive the discriminant negative.

Note that we do not include the case $r_{+} \geq 0, r_{-}<$ 0 since if $r_{+} \geq 0$, the source must lie in one of the ambiguity regions implying that $r_{\text {- }}$ would be nonnegative (i.e., a contradiction). We also disregard the case that both roots are negative since a physical source must lie a nonnegative distance from sensor $i=1$.

\section{Sample storm analyses}

We have applied the LP and QP algorithms (with Mapping \#2) to retrieve several thousand cloud-toground flashes that occurred over the MCTEX analysis region during 28 November (day 332) and 29 November (day 333) 1995. To demonstrate the internal consistency between various forms of the algorithms and to clarify to what degree the algorithms differ, we have analyzed only those flashes that produced a valid excitation at all 

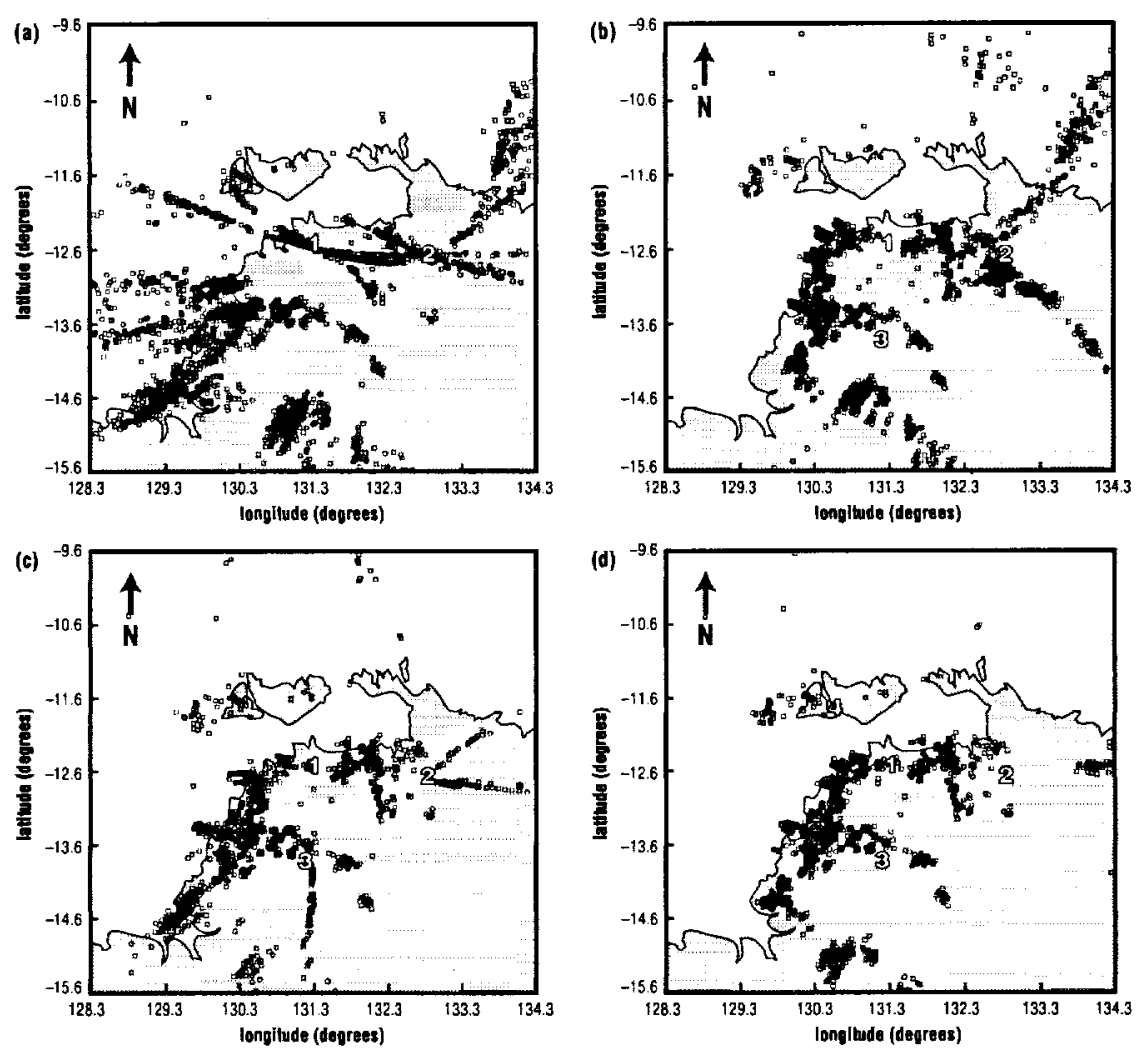

FIG. 13. Day 332 ground flash retrievals using the following algorithms: (a) LP2, (b) LP3, (c) QP, and (d) LP4. Only those source retrievals falling inside the above MCTEX region are shown. Of the four algorithms, the results of LP4 are considered the best estimate of the true lightning locations.

four of the ALDF sites. In this way, each flash can be analyzed by each selected algorithm.

The LP two-station algorithm, or "LP2" algorithm ingests the arrival time and bearing data from sites 1 and 2 , and ignores the data from sites 3 and 4 . The LP3 algorithm ingests the arrival time and bearing data from sites 1, 2, and 3, and ignores site 4. The QP algorithm ingests only arrival time data from sites 1,2 , and 3 , and ignores site 4. Finally, the LP4 algorithm ingests arrival time and bearing data from all four sensors. Hence, the results derived from LP4 are considered to be the best retrieval results against which the three remaining algorithms are compared. For example, the discrepancy between LP2 and LP4 results indicates how appropriate it might be (in future storm analyses) to use LP2 when only two sites detect the flash.

Figure 13 shows the ground flashes derived from day 332 for each algorithm, and Fig. 14 shows the results derived from day 333 . Note from the LP2 results provided in Figs. 13a and 14a that there are obviously location errors along the baseline between site 1 and 2 , in agreement with the general error results provided in section 3 . The cluster of flashes to the south on day 332 (Fig. 13d) is reasonably well retrieved by LP2 (Fig. 13a) since the cluster is not near the baseline, but it is some- what smeared out and biased closer to the network. Similarly, the myriad of flash clusters to the south on day 333 (Fig. 14d) are reasonably well retrieved by LP2 (Fig. 14a), but again there is positional smearing.

The results of LP3 (Figs. 13b and 14b) dramatically reduce the baseline errors and smearing that was associated with the LP2 results. However, there are still some positional adjustments between the LP3 and LP4 results.

Finally, the results of the QP algorithm are shown in Figs. $13 \mathrm{c}$ and $14 \mathrm{c}$. In all of the QP results shown here, root ambiguities were resolved using the LP3 source solutions. Generally, these results agree favorably with the LP4 results and are perhaps even better correlated to the LP4 results than are the LP3 results, for some source locations. However, several sources incorrectly fall along an arc-like pattern that closely resembles the boundaries of the ambiguity region provided in Fig. 10. In the ambiguous case, the simulations of section $6 \mathrm{a}$ also showed us that one solution occurs near the boundary of the ambiguity region and the other well inside the (same) ambiguity region. Because of measurement/ baseline errors and our planar assumptions, LP3 did not always pick the correct root. Hence, sources occurring within the southern cluster (the solution associated with 

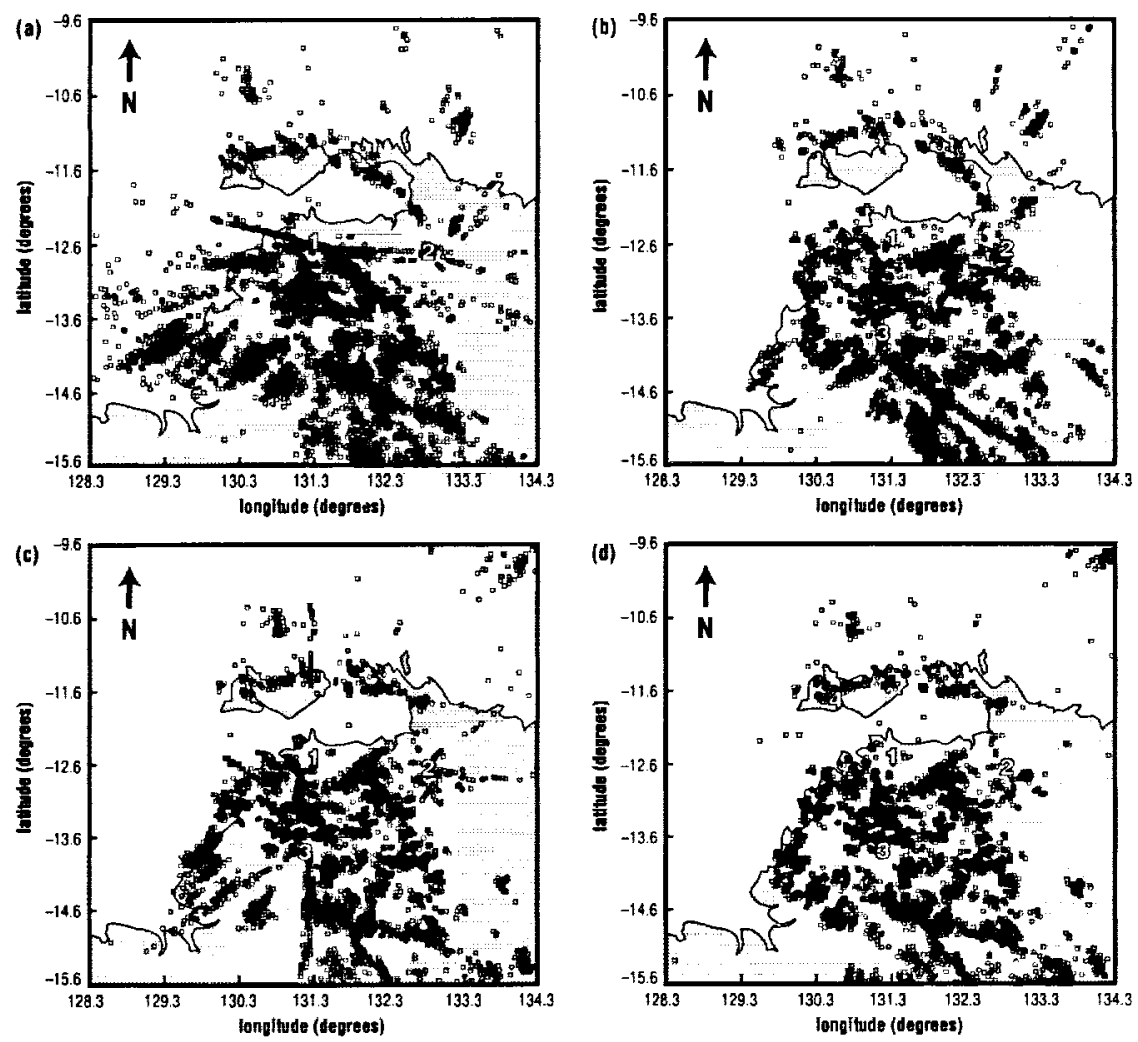

FIG. 14. Day 333 ground flash retrievals using the following algorithms (see algorithm descriptions in text): (a) LP2, (b) LP3, (c) QP. and (d) LP4. Only those source retrievals falling inside the above MCTEX region are shown. Of the four algorithms, the results of LP4 are considered the best estimate of the true lightning locations.

the correct root) had retrieved locations near the boundary of the ambiguity region (the solution associated with the incorrect root). (Note in fact that the southern cluster of Fig. 13c consists of far fewer flashes than in Figs. $13 \mathrm{a}, \mathrm{b}, \mathrm{d}$ indicating that there has been a shift of solutions from the cluster to the border.) In addition, the increase in retrieval errors near the outer sensor baselines (see Fig. 9b) are large and also play a role in defining how well the cluster position is retrieved. Finally, sources very near the outer sensor baselines created complex roots (no solutions). Because the LP4 and QP methods are significantly different mathematical approaches, it is particularly encouraging to see such good agreement between them.

\section{Summary}

In this writing we have derived, tested, and applied two basic methods for retrieving the location and time of occurrence of lightning ground strikes from a network of ALDF sensors. We developed the methods so that they could be used in future validation studies of NASA space-borne lightning sensors: the OTD and the LIS. Because these imagers have a nadir resolution of 8 and $4 \mathrm{~km}$, respectively, our retreival algorithms are adequate for validating lightning source locations within a few hundred kilometers of the ALDF network. As such, we have avoided the need to use the (proprietary) nonlinear $\chi^{2}$ minimization algorithm mentioned in Cummins et al. (1993, 1995, 1998). The development of our algorithms has also expanded upon and clarified the theoretical aspects of ALDF data inversions.

The first approach introduced in this writing, or LP method, assumes that arrival time, bearing, and field amplitude measurements are all available from the network. As provided in (12), these measurements are collected into one coherent linear system of equations that is solved by straightforward inversion. Because of the general form of (12), we have clarified what solution options one has if only a subset of the measurements is available (as when sensors do not trigger on a distant or low amplitude event and/or when sensors are defective). When only three arrival time measurements are available, the LP method cannot be used. To solve this problem, we have introduced a second approach, or QP method.

Both the LP and QP methods described in this writing have problems for sources located near or on the outer sensor baselines. In the case of bearing data, the bearing lines-of-site intersect at small angles, and this fact gives 
rise to large location retrieval error. In the case of timing data, the algebraic solution space is defined geometrically in terms of the intersection of circles (or equivalently, as hyperbolic branches). For sources located near or on the outer sensor baseline, the circles (or hyperbolic branches) intersect at small angles, and again give rise to large location retrieval error. For three- and fourstation networks, the most accurate solutions are found in the region bounded by the three outermost sites. For two-station networks, the most accurate solutions are found outside the line segment joining the two sensors. For all networks, location retrieval error increases with source range.

Since a lightning flash need not trigger all sensors in a given network of $n$ sensors, all of the solution techniques we have described need to be considered. For our four-sensor MCTEX network, we have found that it is best to use LP4 when all four sensors trigger on a discharge. If only three sensors trigger on a flash, we use the QP method (unless the flash is located near an outer sensor baseline, in which case we would employ the LP3 method). LP3 solutions would sometimes be favored over those QP solutions that align along characteristic "arc patterns" such as those shown in Figs. $13 \mathrm{c}$ and $14 \mathrm{c}$. Finally, we employ LP2 when only two sensors detect a flash; because this method has difficulty when the flash is located anywhere along a line passing through the two sensors, we will consider constraining the solution with signal amplitude and/or other ancillary datasets.

The planar methods express the source locations directly in terms of the measurements. The solutions are concise, require little computer time, and afford the user with specific physical insights about the retrieval problem in the form of analytic equations. The equations express the relative importance and effects of timing/ bearing data on the accuracy of final solutions. They also describe the regions of ambiguity, and the regions where source locations produce complex roots.

Moreover, the LP and QP methods introduced here offer the authors and other researchers a means to intensively analyze and compare, first hand, lightningradio-source locations with OTD-LIS low-earth orbit lightning detections. In the future, we intend to apply these methods to analyze a wide range of thunderstorms, to continue intercomparing the methods, and to relate the results to OTD and LIS and other independent datasets such as radar, Lightning Detection and Ranging (LDAR), and the National Lightning Detection Network (NLDN). The first author will also improve some of the matrix methods presented here to directly account for earth sphericity; more elegant oblate spheroidal models are also under consideration.

Acknowledgments. We appreciate the work of Susan Burrer and Janine Roskowski for their help in preparing the final manuscript and figures. In addition, we are thankful to Mike Stewart, David Slaton, Jim Core, Steven Rutledge, Steven Tirus, and other MCTEX personnel for their assistance in the deployment, testing, and maintenance of the ALDF sites that stimulated the theoretical work provided in this writing. Finally, we are grateful for the support of this research by Dr. Ramesh Kakar, manager of the Atmospheric Dynamics and Remote Sensing Program at NASA Headquarters, under Task \#622-29-40, to the NASA's Earth Observing System (EOS) Program under Task \#229-71-46, and to Dr. James Dodge and David O'C. Starr under NASA Grant NAG65-6461.

\section{APPENDIX A}

\section{Weighting of Bearing Data}

It is of interest to determine to what extent bearing data is actually being used to constrain the lightning source location in the LP method as a function of the weighting factor $\delta$ introduced into the linear system of equations provided in (11). Insight is gained by considering the case of three sensors and ignoring field measurements, $F$. Then (11) reduces to

$$
\left[\begin{array}{c}
\alpha_{2} \\
\alpha_{3} \\
\delta \beta_{1} \\
\delta \beta_{2} \\
\delta \beta_{3}
\end{array}\right]=\left[\begin{array}{ccc}
\left(x_{2}-x_{1}\right) & \left(y_{2}-y_{1}\right) & c\left(t_{1}-t_{2}\right) \\
\left(x_{3}-x_{1}\right) & \left(y_{3}-y_{1}\right) & c\left(t_{1}-t_{3}\right) \\
\delta \sin \phi_{1} & -\delta \cos \phi_{1} & 0 \\
\delta \sin \phi_{2} & -\delta \cos \phi_{2} & 0 \\
\delta \sin \phi_{3} & -\delta \cos \phi_{3} & 0
\end{array}\right]\left[\begin{array}{c}
x \\
y \\
d_{1}
\end{array}\right] .
$$

This system, which can be written in the standard notation $\mathbf{g}=\mathbf{K} \mathbf{f}$, has the least squares solution $\mathbf{f}=(\tilde{\mathbf{K}} \mathbf{K})$ ' $\tilde{\mathbf{K}} \mathbf{g}$. We are interested in the explicit functional dependence of $x$ and $y$ on the arrival time $t_{i}$ and bearing $\phi_{i}$ data, and on the weighting factor $\delta$. Because this is a very involved hand calculation, we utilize a computer-aided symbolic manipulator to arrive at the following form:

$x=\frac{1}{h_{0} \delta^{2}+h_{1}}\left[h_{2} \alpha_{2}+h_{3} \alpha_{3}+\sum_{j-4}^{6}\left(h_{j} \delta^{2}+h_{j+3}\right) \beta_{j 3}\right]$.

The 10 functions, $h_{j}(j=0, \ldots, 9)$ depend, in general, on the arrival time and bearing data. The variables $\left(\alpha_{2}\right.$, $\alpha_{3}$ ) depend only on arrival time data and network geometry, and the variables $\left(\beta_{1}, \beta_{2}, \beta_{3}\right)$ depend only on bearing data and network geometry as given in (3) and (6), respectively. A similar form holds for $y$. The coefficient in front of the square brackets in (A2) does not preferentially weight the $\alpha$ 's or $\beta$ 's so it is of no concern in this discussion. However, the coefficients $\left(h_{j}+\right.$ $\delta^{2} h_{j+3}$ ) do weight the $\beta$ 's but not the $\alpha^{\prime}$ 's (i.e., these coefficients weight the bearing data, but not the arrival time data). This leads to the final results 


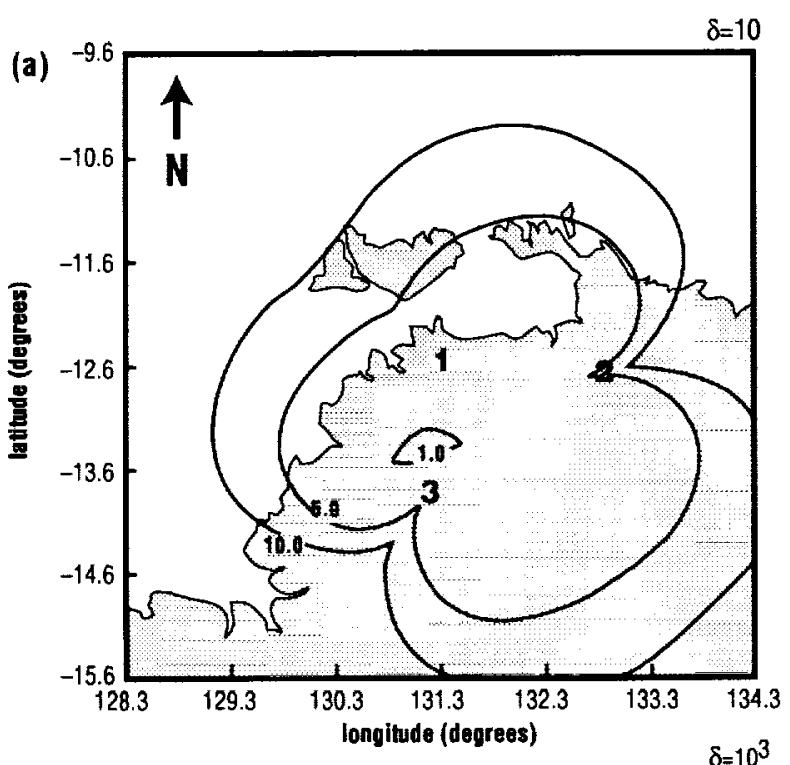

(b)
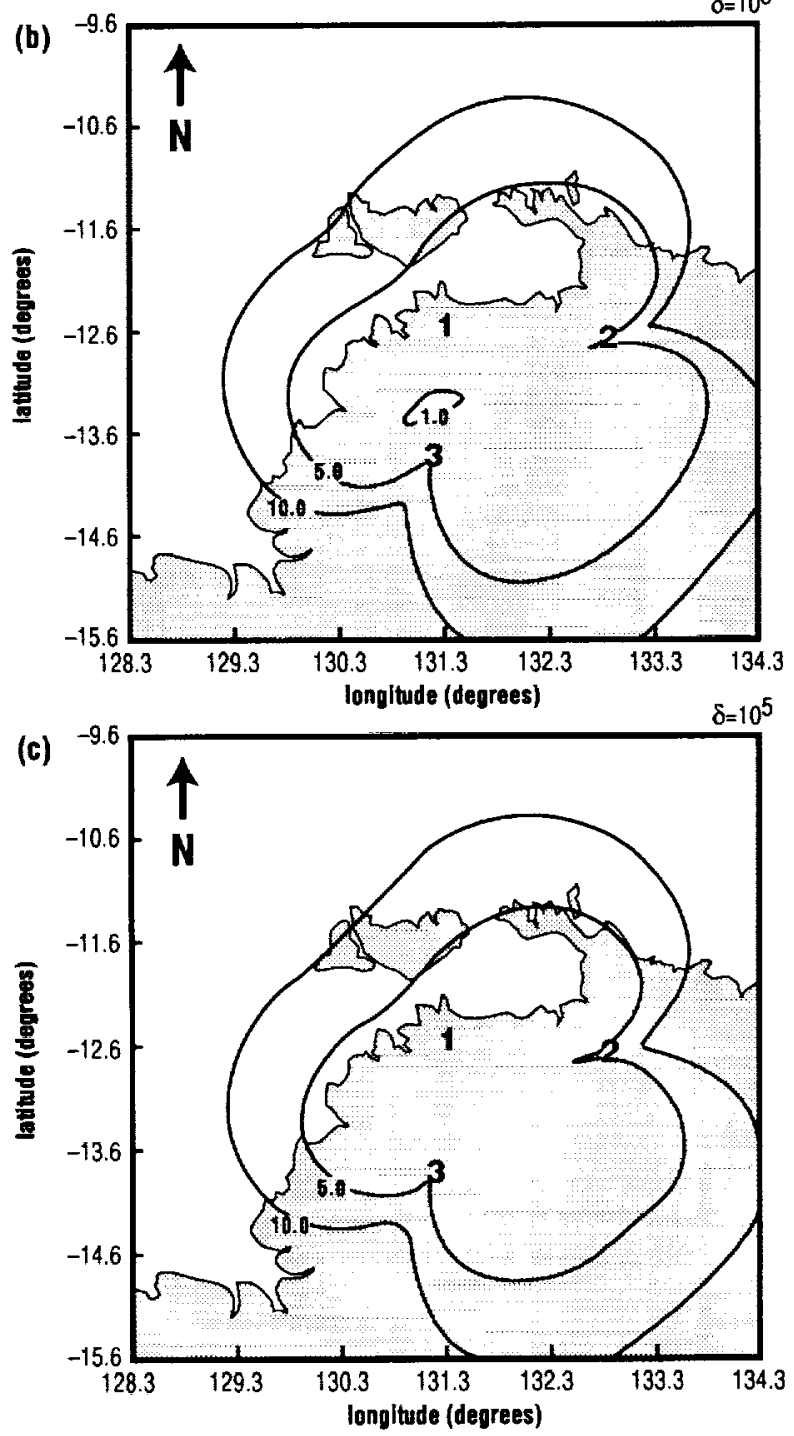

$$
\begin{gathered}
\delta=0 \Rightarrow \text { no solution } \\
\lim _{\delta \rightarrow \pi} x=\frac{1}{h_{0}}\left(h_{4} \beta_{1}+h_{5} \beta_{2}+h_{6} \beta_{3}\right) \equiv \Lambda\left(\phi_{i}\right) ; \\
i=1,2,3 .
\end{gathered}
$$

The first result is true since when $\delta=0$ there are two equations in three unknowns in (Al). The second result is true because the only arrival time dependence associated with each function $\left(h_{0}, h_{4}, h_{5}, h_{6}\right)$ is a factor $\left(t_{2}^{2}+t_{3}^{2}\right)$. When the ratios $h_{1} / h_{0}$ are taken, this factor cancels out and we are left with a function, $\Lambda$, that depends only on bearing data.

In summary, for a positive finite value of $\delta$, both arrival time and bearing data are utilized. However, as $\delta$ is increased from zero, bearing data eventually becomes more heavily weighted over arrival time data until, for a sufficiently large value of $\delta$, only bearing data is being used to determine the source location. For $\delta=1$, we have difficulty inverting $\mathbf{K}$ for many source locations. We have also performed retrievals, in the presence of measurement errors, for the values: $\delta=10,10^{2}$, $10^{7}, 10^{4}, 10^{5}$, and $10^{6}$. Figure Al shows the result for $\delta=10,10^{3}$, and $10^{5}$. There is not much change in the solution from 10 to $10^{3}$, but the dominance of bearing data constraints at $10^{5}$ and higher begins to reduce the quality of the solution (i.e., a $2^{\circ}$ random bearing error can create a substantial location error if the source range is sufficiently large).

We have performed the same type of computer-aided symbolic manipulation to determine explicit forms when a four-sensor network is used [i.e., one more arrival time equation and one more bearing equation are added to the system in (A1) so that $\mathbf{K}$ becomes a $7 \times$ 3 matrix ]. In this case, the form of $x$ (as well as $y$ ) is

$$
\begin{aligned}
x= & \frac{1}{k_{0} \delta^{4}+k_{i} \delta^{2}+k_{2}} \\
& \times\left[\sum_{j=2}^{4}\left(k_{j+i} \delta^{2}+k_{j+4}\right) \alpha_{j}+\sum_{j=1}^{4}\left(k_{j+8} \delta^{4}+k_{j+12} \delta^{2}\right) \beta_{j}\right] .
\end{aligned}
$$

Most of the 17 functions, $k_{i}, j=0, \ldots, 16$ depend on both arrival time and bearing data. However, $\left(k_{2}, k_{6}, k_{7}\right.$, $k_{\mathrm{b}}$ ) depend on arrival time data, but do not depend on bearing data. We obtain the final limiting conditions

FIG. A I. Location retrieval errors for a three-station network using the LP method when (a) $\delta=10$, (b) $\delta=10^{3}$, and (c) $\delta=10^{5}$. The same simulated measurement errors discussed in section 3 are used: $0.5-\mathrm{m}$ sensor location crror, $300-\mathrm{ns}$ timing error, $2^{\circ}$ bearing crror. Mapping \#2 is used. Contours $(\mathrm{km})$. 
$\lim _{\delta \rightarrow 0} x=\frac{1}{k_{2}}\left(k_{6} \alpha_{2}+k_{7} \alpha_{3}+k_{8} \alpha_{4}\right) \equiv \Gamma\left(t_{i}\right)$

$\lim _{\delta \rightarrow \infty} x=\frac{1}{k_{0}}\left(k_{\iota} \beta_{1}+k_{10} \beta_{2}+k_{11} \beta_{3}+k_{12} \beta_{4}\right) \equiv \Omega\left(\phi_{i}\right) ;$

$$
i=1,2,3 \text {. }
$$

Hence, we swing from a solution governed only by timing data $(\delta=0)$ to one governed only by bearing data $(\delta=\infty)$. The solutions for $\delta=10,10^{3}$, and $10^{5}$ are shown in Fig. A2. The bearing data significantly worsens the solution when weighted heavily $\left(\delta=10^{5}\right)$.

\section{APPENDIX B}

\section{Locations Where QP Method Discriminant Function Vanishes}

We investigate more rigorously the zeroes of the discriminant function $\Delta \equiv\left(B^{2}-4 A C\right)$ of $(27)$ in the QP method. The computer plots of this function gave some interesting results near the outer baselines of the sensors, that is, there appears to be minima there (see Fig. 11).

In the following formal approach, we algebraically reduce the discriminant into the product of three factors. Each factor is then shown to vanish along a specific outer sensor baseline. Our results apply to arbitrary network geometries. Because a zero discriminant implies that two nonnegative, real, and equal roots are obtained, a unique (unambiguous) solution $[x(r), y(r), t(r)]$ is obtained on the outer sensor baselines where $r=-B /(2 A)$ $=r_{+}=r_{-}$.

Using the forms in (26) for $A, B$, and $C$, the discriminant can be written as

$$
\begin{gathered}
\Delta=4 c^{2}\left[\left(p^{2}-r_{2}^{2} r_{3}^{2}\right)\left(q_{2}^{\prime 2} t_{3}^{2}-2 q_{2}^{\prime} q_{3}^{\prime} t_{2} t_{3}+q_{3}^{\prime 2} t_{2}^{2}\right)\right. \\
\left.+\varepsilon^{2} \sigma\right],
\end{gathered}
$$

where

$$
\begin{aligned}
p & =\mathbf{r}_{2} \cdot \mathbf{r}_{3}=x_{2} x_{3}+y_{2} y_{3} \\
\boldsymbol{\varepsilon} & =\frac{1}{c} \operatorname{det} \mathbf{Q}=\frac{1}{c}\left(x_{2} y_{3}-y_{2} x_{3}\right) \\
\sigma & =r_{3}^{2} q_{2}^{\prime 2}-2 p q_{2}^{\prime} q_{3}^{\prime}+r_{2}^{2} q_{3}^{\prime 2} .
\end{aligned}
$$

To simplify some of the algebra without losing generality, we rotate the $x$ and $y$ axes so that $y_{2} \equiv 0$. Further reduction of (B1) leads to

$$
\begin{aligned}
\Delta= & {\left[x_{2}^{2} y_{3}^{2}\left(x_{2}^{2}-c^{2} t_{2}^{2}\right)\right]\left[r_{3}^{2}-c^{2} t_{3}^{2}\right] } \\
& \times\left[\left(x_{2}^{2}-c^{2} t_{2}^{2}\right)+2\left(c^{2} t_{2} t_{3}-x_{2} x_{3}\right)+\left(r_{3}^{2}-c^{2} t_{3}^{2}\right)\right] .
\end{aligned}
$$

Figure B 1 considers the second factor, $\left(r_{3}^{2}-c^{2} t_{3}^{2}\right)$, in (B3). For a source located on the solid line with $r \geq r_{3}$ site three is excited at
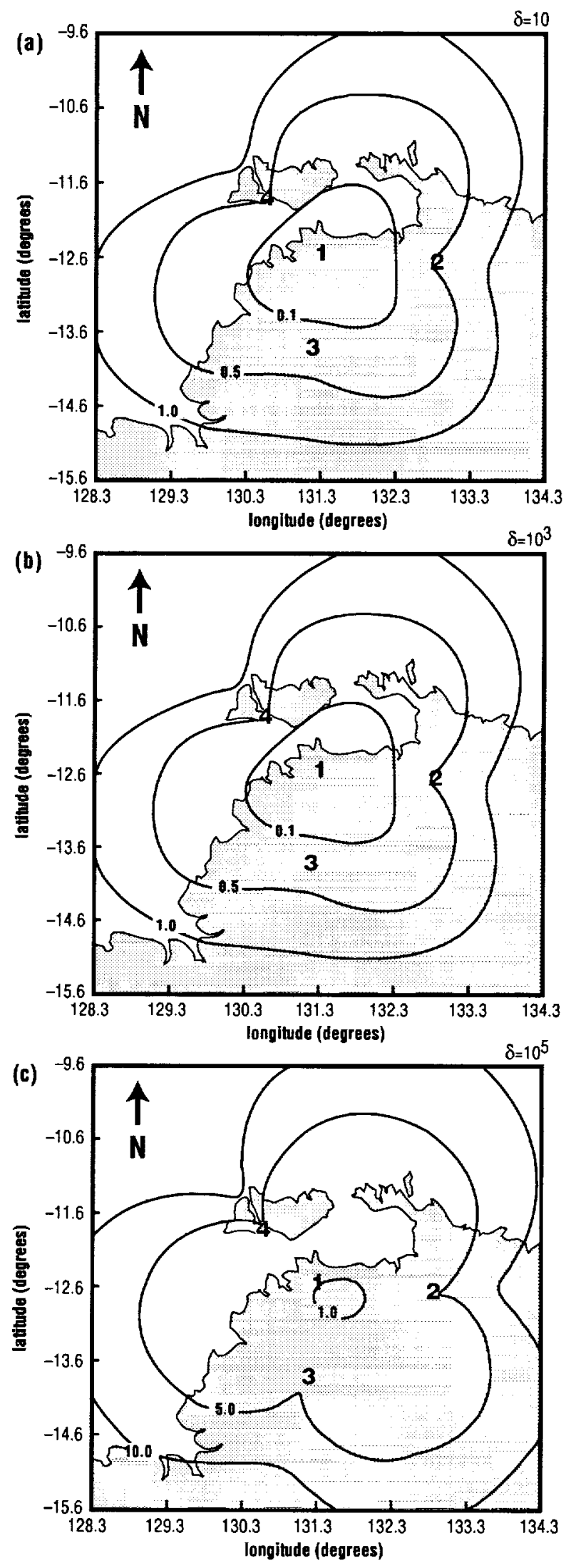

FIG. A2. Same as in Fig. A1, but for a four-station network. 


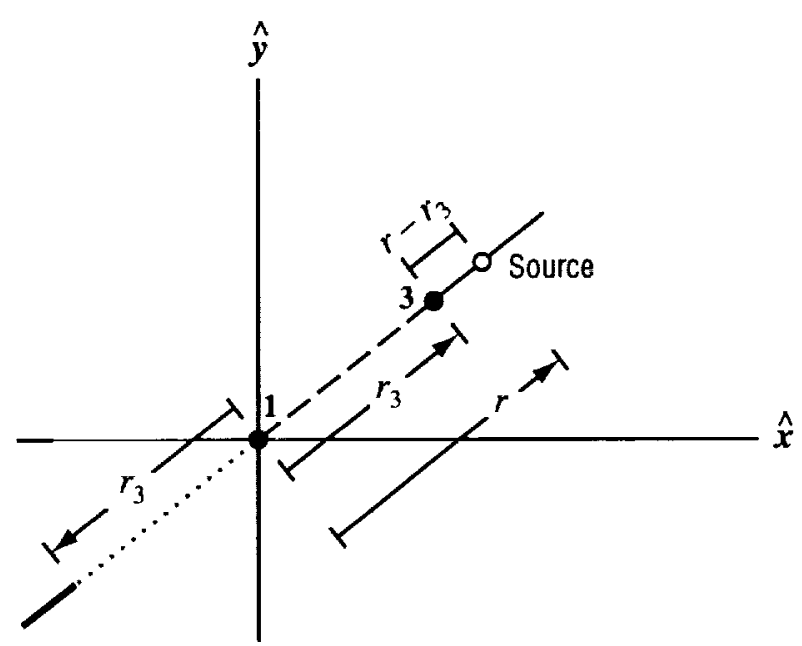

FIG. B 1. Geometry for analyzing the second factor in the discriminant function.

$$
t_{3}=t+\frac{r-r_{3}}{c}=\frac{-r}{c}+\frac{r-r_{3}}{c}=-\frac{r_{3}}{c} .
$$

The factor becomes

$$
\left(r_{3}^{2}-c^{2} t_{3}^{2}\right)=r_{3}^{2}-c^{2}\left(-\frac{r_{3}}{c}\right)^{2}=r_{3}^{2}-r_{3}^{2}=0 .
$$

Proceeding in a similar fashion, the factor for a source on the dashed line with $0<r<r_{3}$ is the nonzero result, $4 r\left(r_{3}-r\right)$, and the factor for a source on the dotted line (with $0 \leq r<r_{3}$ ) or a source on the thick line (with $r$ $\geq r_{3}$ ) is zero. Hence, the factor is zero along the line running through the sensors (including the sensor locations themselves, but excluding the line segment between the sensors). This is what we refer to as the "outer sensor baselines." Similar comments can be made regarding sites 1 and 2 when the factor $\left(x_{2}^{2}-c^{2} t_{2}^{3}\right)$ is considered.

Evidently the third factor in (B3) corresponds to the line running through sites 2 and 3 . To prove this, we consider the geometry provided in Figure B2. For a source on the solid line with $d \geq 0$ we have

$$
t_{2}=\frac{D+d-r}{c} \quad t_{3}=\frac{d-r}{c} .
$$

Substituting these expressions into the third factor, and noting that $D^{2}=\left(x_{2}-x_{3}\right)^{2}+y_{3}^{2}=x_{2}^{2}-2 x_{2} x_{3}+r_{3}^{2}$, we obtain

$$
\begin{aligned}
x_{2}^{2}- & (D+d-r)^{2}+r_{3}^{2}-(d-r)^{2} \\
+ & 2(D+d-r)(d-r)-2 x_{2} x_{3} \\
= & \left(x_{2}^{2}-2 x_{2} x_{3}+r_{3}^{2}\right)-[(D+d-r)-(d-r)]^{2} \\
= & \left(x_{2}^{2}-2 x_{2} x_{3}+r_{3}^{2}\right)-D^{2}=0 .
\end{aligned}
$$

For a source located on the dashed line (but not at site

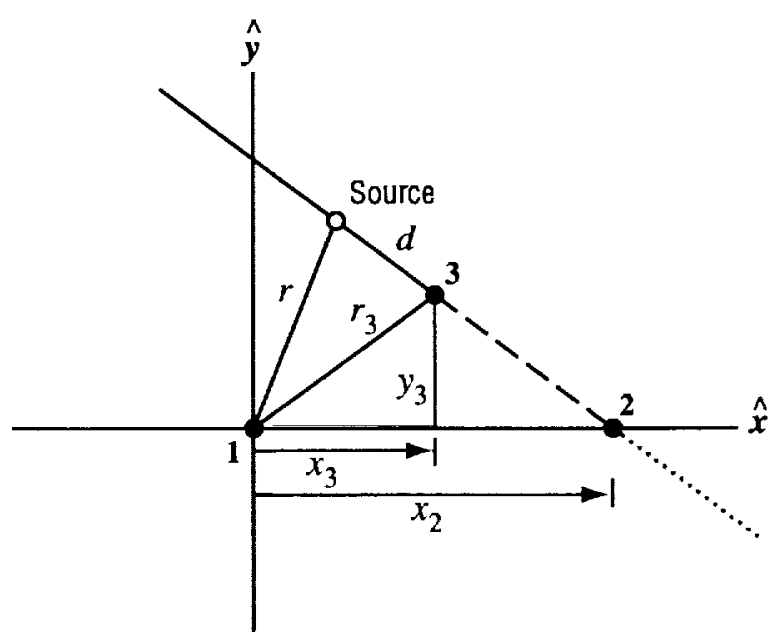

FIG. B2. Geometry for analyzing the third factor in the discriminant function.

2 or site 3) the factor reduces to the nonzero result: $4 \omega_{2} \omega_{3}\left[\left(x_{2}-x_{3}\right)^{2}+y_{3}^{2}\right]$, where the constant factors $\left(\omega_{2}\right.$, $\left.\omega_{3}\right)$ obey the constraints $\omega_{2}+\omega_{3}=1, \omega_{2}>0, \omega_{3}>$ 0 . Finally, for a source on the dotted line and a distance $l \geq 0$ from site 2 , we have

$$
t_{2}=\frac{l-r}{c} \quad t_{3}=\frac{D+l-r}{c} .
$$

These expressions have the same form as those in (B6) but are interchanged. When substituted into the third factor of (B3), the factor reduces to zero as in (B7). This completes the proof showing that the discriminant function vanishes along the outer sensor baselines.

\section{REFERENCES}

Christian, H. J., R. J. Blakeslee, and S. J. Goodman, 1992: Lightning Imaging Sensor (LIS) for Earth Observing System. NASA Tech Memo. TM-4350, $36 \mathrm{pp}$.

— S. J. Goodman, R. J. Blakeslee, W. J. Koshak, D. M. Mach, and W. L. Boeck, 1995: The Optical Transient Detector: First results. Proc. 1995 IUGG XXI Meeting. Boulder, CO, International Union of Geodesy and Geophysics, A264.

Cooray, V., 1987: Effects of propagation on the return stroke radiation fields. Radio Sci., 22, 757-768

Cummins, K. L., R. O. Burnett, W. L. Hiscox, and A. E. Pifer, 1993 : Line reliability and fault analysis using the National Lightning Detection Network. Preprints, Precise Measurements in Power Conf., Arlington, VA, National Science Foundation and Center for Power Enginecring at Virginia Tech., II-4.I-IT-4.15.

- E. A. Bardo, W. L. Hiscox, R. B. Pyle, and A. E. Pifer, 1995 NLDN'95: A combined TOA/MDF technology upgrade of the U.S. National Lightning Detection Network. Int. Aerospace and Ground Conf. on Lightning and Static Electricity, Williamsburg. VA, National Interagency Coordination Group, 72-1-72-15.

- M. J. Murphy, E. A. Bardo, W. L. Hiscox, R. B. Pyle, and A. E. Pifer, 1998: A combincd TOAMDF technology upgrade of the U.S. National Lightning Detection Network. J. Geophys. Res., 103 (D8), 9035-9044.

Hiscox, W. L., E. P. Krider, A. E. Pifer, and M. A. Uman, 1984: A systematic method for identifying and correcting "site errors" 
in a network of magnetic direction finders. Preprints, Int. Aerospace and Ground Conf. on Lightning and Static Electricity, Orlando, FL, National Interagency Coordination Group, 7- 1-7-5.

Holle, R. L., and R. E. Lopez, 1993: Overview of real-time lightning detection systems and their metcorological uses. NOAA Tech. Memo. ERL NSSL-102, 73 pp.

Honma, N., F. Suzuki, Y. Miyake, M. Ishii, and S. Hidayat, 1998: Propagation effect on field waveforms in relation to time-ofarrival technique in lightning location. J. Geophys, Res. 103 (DI2), I4 I41-14 I45.

Kcenan, T., and Coauthors, 1994: Science Plan Maritime Continent Thunderstorm Experiment (MCTEX). BMRC Research Rep. 44, $61 \mathrm{pp}$. [Available from Bureau of Metcorology Research Centre, BMRC GPO Box 1289K, Melbourne, Victoria, Australia 3001.]

-, R. Carbone, S. Rutledge, J. Wilson, G. Holland, and P. May, 1996: The Maritime Continent Thunderstorm Experiment (MCTEX): Overview and initial results. Preprints, Seventh Conf. on Mesescale Processes, Reading, United Kingdom, Amer. Meteor. Soc., 326-328.
Koshak, W. J,, and E. P. Krider, 1994: A linear method for analyzing lightning field changes. $J$. Atmos. Sci., 51, 473-488.

- , and R. J. Solakiewicz, 1996: On the retricval of lightning radio sources from time-of-arrival data. J. Geophys. Res., 101, 2663126639.

Orville, R. E., 1987: An analytic solution to obtain the optimum source Iocation using multiple direction finders on a spherical surface. J. Geophys. Res., 92, $10877-10886$ , and Coauthors, 1997: Lightning in the region of the TOGA COARE. Bull. Amer. Meteor. Soc, 78, 1055-1067.

Peterson, W. A., S. A. Rutledge, and R. E. Orville, 1996: Cloud-toground lightning observations from TOGA COARE: Selected results and lightning location algorithms. Mom. Wea. Re' ', 124, $602-620$.

Twomey, S. A., 1997: Introduction to the Mathenatics of Inversion in Remote Sensing and Indirect Measurements. Elsevier, 243 pp.

Uman, M. A., D. K. McLain, R. J. Fisher, and E. P. Krider, 1973 Electric field intensity of the lightning return stroke. J. Geophys. Res., 78, 3523-3529. 
